\title{
On the dynamics of proto-neutron star winds and r-process nucleosynthesis
}

\author{
I. V. Panov ${ }^{1,2}$ and H.-Th. Janka ${ }^{1}$ \\ 1 Max-Planck-Institut für Astrophysik, Karl-Schwarzschild-Straße 1, 85748 Garching, Germany \\ 2 Institute for Theoretical and Experimental Physics, B. Cheremushkinskaya 25, Moscow 117218, Russia \\ e-mail: Igor.Panov@itep.ru
}

Received 30 May 2008 / Accepted 26 November 2008

\begin{abstract}
We study here the formation of heavy r-process nuclei in the high-entropy environment of rapidly expanding neutrino-driven winds from compact objects. In particular, we explore the sensitivity of the element creation in the $A \gtrsim 130$ region to the low-temperature behavior of the outflows. For this purpose we employ a simplified model of the dynamics and of the thermodynamical evolution for radiation dominated, adiabatic outflows. It consists of a first stage of fast, exponential cooling with timescale $\tau_{\mathrm{dyn}}$, followed by a second phase of slower evolution, assuming either constant density and temperature or a power-law decay of these quantities. These cases describe a strong deceleration or decreasing acceleration of the transsonic outflows, respectively, and thus are supposed to capture the most relevant effects associated with a change in the wind expansion behavior at large radii, for example because of the collision with the slower, preceding supernova ejecta and the possible presence of a wind termination shock. We find that for given entropy, expansion timescale, and proton-to-baryon ratio not only the transition temperature between the two expansion phases can make a big difference in the formation of the platinum peak, but also the detailed cooling law during the later phase. Because the nuclear photodisintegration rates between about $2 \times 10^{8} \mathrm{~K}$ and roughly $10^{9} \mathrm{~K}$ are more sensitive to the temperature than the neutron-capture rates are to the free neutron density, a faster cooling but continuing high neutron density in this temperature regime allow the r-process path to move closer to the neutron-drip line. With low $(\gamma, \mathrm{n})$ - but high $\beta$-decay rates, the r-processing does then not proceed through a $(\gamma, \mathrm{n})$-(n, $\gamma)$ equilibrium but through a quasi-equilibrium of $(\mathrm{n}, \gamma)$-reactions and $\beta$-decays, as recently also pointed out by Wanajo. Unless the transition temperature and corresponding (free neutron) density become too low $\left(T \lesssim 2 \times 10^{8} \mathrm{~K}\right)$, a lower temperature or faster temperature decline during the slow, late evolution phase therefore allow for a stronger appearance of the third abundance peak.
\end{abstract}

Key words. nuclear reactions, nucleosynthesis, abundances - stars: supernovae: general - stars: winds, outflows - stars: neutron

\section{Introduction}

Heavy nuclei beyond the iron peak are known to be produced in nature mainly through neutron capture reactions (Burbidge et al. 1957). Rapid neutron capture and the reverse photodisintegration processes achieve an equilibrium among the isotopes of each heavy element. Then beta-decay occurs that leads to the increase in the nuclear charge and formation of a new element. When the neutron capture rate is much higher than the beta-decay rate $\left(\lambda_{\mathrm{n} \gamma}>\lambda_{\beta}\right)$ and $T_{9} \sim 1\left(T_{9}\right.$ is defined as the temperature normalized to $10^{9} \mathrm{~K}$ ), the r-process can start and, for a sufficiently high neutron-to-seed ratio, the wave of nucleosynthesis drives the process to heavier nuclei, forming, in part, the abundance r-process peaks at $\sim 80,130$ and 196.

Although the r-process sites remain unknown, many astrophysical models and sources for r-process elements have been proposed during the past 50 years, including, in particular, scenarios such as that of an explosion on a neutron-star surface (Bisnovatyi-Kogan \& Chechetkin 1979), a collision of a neutron star with a black hole (Lattimer \& Schramm 1976), an explosion of a low-mass neutron star (Imshennik 1992), and the hypothetical escape of nucleon bubbles in case of a soundless stellar collapse (Imshennik \& Litvinova 2006). The last scenario was suggested as the origin of gamma-ray bursts but could also be an interesting site for the nucleosynthesis of heavy elements.
Presently, however, it seems most likely that rapid n-capture nucleosynthesis can take place during different stages of supernova explosions (e.g. Hillebrandt 1978; Woosley \& Hoffman 1992), or in neutron star mergers (e.g. Lattimer \& Schramm 1974; Symbalisty \& Schramm 1982; Freiburghaus et al. 1999). An overview of the currently discussed possible sites is given in a recent review paper by Arnould et al. (2007).

Supernovae and neutron star mergers have different advantages and weak points, but the main difference lies probably in the initial neutron-to-proton ratio, which is necessary for supporting a sufficiently high free neutron density during several hundreds of milliseconds. With respect to this parameter, all astrophysical scenarios can be separated into two distinct groups, in which nucleosynthesis can be carried out over short or long timescales (so-called short-time or long-time solutions, respectively), as introduced by Seeger et al. (1965). In nature, the r-process might be realized in both these types of sources and also other ones might contribute (see for example Cameron 2002; Arnould et al. 2007). It is only future modeling and observations that will be able clarify the multiplicity of the r-process models and types, as well as the contributions from different production sites to the r-process element abundances observed in the Solar System.

Among the astrophysical events proposed as sites for the r-process, supernova explosions still remain the preferable ones (see, e.g. Wanajo \& Ishimaru 2006, and references therein). 
In particular, supernova explosions can distribute r-process material all over the Galaxy, and estimated amounts of heavy elements produced in $\mathrm{SN}$ explosions are in accordance with the observations.

The neutrino-driven wind from a hot neutron star produced in a supernova explosion has been considered as a probable site for the r-process by many authors (see e.g. Meyer et al. 1992; Woosley et al. 1994; Witti et al. 1993; Otsuki et al. 2000; Sumiyoshi et al. 2000; Terasawa et al. 2001; Wanajo et al. 2001). A part of the surface material of a neutron star is heated by the supernova neutrinos and gets ejected. It can be described as a hot outflow with a fairly high entropy and a moderate density.

Various studies of the r-process element formation in protoneutron star winds have been conducted during the past years. Hoffman et al. (1997), employing the analytic wind model of Qian \& Woosley (1996), explored the possibility of third peak creation for different combinations of the determining parameters of $Y_{\mathrm{e}}$ (the electron-to-baryon ratio), entropy, and expansion timescale. Altogether, they showed (see their Fig. 10) that for typical values of $Y_{\mathrm{e}} \gtrsim 0.4$ in the wind the third r-process peak can be produced for combinations ranging from moderate entropy ( $s \sim 100$ in units of Boltzmann's constant per baryon) and very short timescales ( $t_{\exp }$ a few milliseconds) to high entropies $(s \gtrsim 400)$ and long expansion timescales (a few $100 \mathrm{~ms})$. The wind models existing at that time failed to provide the necessary conditions (Witti et al. 1994; Qian \& Woosley 1996).

Subsequently, several other studies of neutrino-driven winds in the framework of general relativistic gravity (Otsuki et al. 2000; Sumiyoshi et al. 2000; Thompson et al. 2001; Wanajo et al. 2001, 2002) confirmed the need of fairly extreme conditions concerning expansion timescale or entropy for strong r-processing up to $A \sim 200$. Since very high entropies could not be obtained in the wind scenario or were found to be associated with too low mass loss rates for any significant production of $\mathrm{r}$ nuclei, these studies also demonstrated a preference for the case of moderate entropies, $s \sim 100-200$, and very short timescales. This seemed to give a bias for winds from compact neutron stars with a large mass, $M \gtrsim 2 M_{\odot}$, and a small radius $R \lesssim 10 \mathrm{~km}$ as the most likely site for r-process element formation up to the platinum peak.

This conclusion was found to hold independent of whether freely expanding, transsonic "wind" outflows were considered (Hoffman et al. 1997; Thompson et al. 2001) or subsonic "breeze" solutions (Otsuki et al. 2000; Sumiyoshi et al. 2000; Wanajo et al. 2001; Terasawa et al. 2001, 2002) or supersonic winds with some fixed freeze-out temperature $T_{\mathrm{f}}$ (Wanajo et al. 2002, Wanajo 2007). Winds are characterized by a monotonically increasing velocity and a continuous temperature decrease when the radius goes to infinity, whereas breezes are obtained when a prescribed, non-vanishing value of the pressure and temperature is required to be reached at the outer boundary at some large radius (this is supposed to mimic the fact that the fast neutrino-driven wind is decelerated again as it collides and merges with the preceding, more slowly expanding material ejected in the early phase of the supernova blast). The approach taken by Wanajo et al. (2002) allowed them to causally disconnect the conditions at large radii from those at the neutron star and thus to choose the asymptotic temperature independent of the mass-loss rate (in contrast to the situation for breezes and winds). The results from these different types of outflow scenarios concerning combinations of entropy, $Y_{\mathrm{e}}$, and expansion timescale necessary for strong $r$-processing in the ejecta turned out to be in qualitative agreement (compare, e.g., Fig. 1 in Thompson et al. 2001 with Fig. 8 in Otsuki et al. 2000 and with Table 2 and Fig. 6 in Sumiyoshi et al. 2000).

Later Terasawa et al. (2002) announced to have found successful conditions even for proto-neutron stars with a more typical mass around $1.4 M_{\odot}$ and a radius of $10 \mathrm{~km}$. Like previous studies (in particular Sumiyoshi et al. 2000, but also Otsuki et al. 2000) they considered breeze outflows, but different from the earlier investigations they chose a lower value for the outer boundary pressure, which implied a lower asymptotic temperature. They argued that this is favorable for a strong r-processing up to the third peak because the lower final temperature is associated with a faster expansion and more rapid cooling. The more quickly decreasing temperature leads to a slowing down of charged-particle reactions and reduces the efficiency of $\alpha$ particle recombination. Therefore it leads to less production of seed nuclei and a higher neutron-to-seed ratio. Such a sensitivity to the expansion timescale was also seen by Arnould et al. (2007), who performed systematic variations of the parameters in analytic wind and breeze solutions. Arnould et al. (2007) verified that wind solutions provide the more favorable conditions for strong r-processing than the slower breezes, because for given values of the entropy and mass loss rate the expansion timescale is directly correlated with the asymptotic value of the temperature and thus of the total specific energy of the outflowing gas. These values are lowest in case of freely expanding winds. Arnould et al. (2007), however, also saw that the mass loss rate has a much more sensitive influence: breeze solutions with higher mass loss rates (and otherwise the same characterising parameter values) make a faster expansion and allow for a stronger r-process despite having higher asymptotic values of the temperature.

Although Arnould et al. (2007) confirmed the formation of the third r-process peak for combinations of entropy, expansion timescale, $Y_{\mathrm{e}}$, neutron star mass, and asymptotic temperature in the ballpark of those considered by Terasawa et al. (2002), the results of the latter paper are nevertheless in contradiction to the earlier studies by Otsuki et al. (2000), Sumiyoshi et al. (2000), and Thompson et al. (2001): Terasawa et al. (2002) obtained a significantly higher entropy and shorter expansion timescale than Sumiyoshi et al. (2000) even for the same choice of outflow determining conditions, i.e., for the same individual neutrino luminosity $\left(L_{v_{i}}=10^{51} \mathrm{erg} \mathrm{s}^{-1}\right)$, the same mean neutrino energies, and in particular the same outer boundary pressure (compare Table 1 in the Terasawa et al. work and Sect. 3.2 in Sumiyoshi et al.). Moreover, for all tested values of the boundary pressure, Terasawa et al. (2002) found outflow properties for their $1.4 M_{\odot}$ neutron star that were largely different from those plotted for breezes in Fig. 8 of Otsuki et al. (2000) and for freely expanding winds in Fig. 11 of Thompson et al. (2001). These differences seem to have been causal for the successful solar system like r-process reported by Terasawa et al. (2002), but the actual reason why the more favorable outflow behavior was obtained, remains unexplained ${ }^{1}$.

Recently, Arcones et al. (2007) performed new hydrodynamic simulations of neutrino-driven winds, systematically exploring the effects of the wind termination shock that forms when the supersonic wind collides with the slower earlier supernova ejecta (Janka \& Müller 1995a,b; Burrows et al. 2005;

\footnotetext{
1 Note that Thompson et al. (2001) used a sophisticated description of the equation of state and thus explicitly accounted for the nonrelativistic character of electrons and positrons at low temperatures, an effect that Sumiyoshi et al. (2000) pointed out to be important for a reliable determination of the expansion timescale.
} 
Buras et al. 2006, see also Tomàs et al. 2004) and that decelerates the outflow abruptly. They found that the position of the reverse shock is strongly dependent on the evolution phase, progenitor structure, and explosion energy of the supernova. Motivated by these studies, interest has recently turned to a closer exploration of the relevance of the late-time wind dynamics for r-process nucleosynthesis. On the one hand, Wanajo (2007) showed numerically that a solar-like r-process can also be produced in supersonically expanding outflows whose temperature drops quickly to a few $10^{8} \mathrm{~K}$ instead of asymptoting to a value around $10^{9} \mathrm{~K}$ as previously mostly assumed (Otsuki et al. 2000; Sumiyoshi et al. 2000; Wanajo et al. 2001, 2002). In such a low-temperature environment an $(\mathrm{n}, \gamma)-(\gamma, \mathrm{n})$ equilibrium is never achieved during the nucleosynthesis of heavy r-process material, but neutron captures compete with $\beta$-decays in the low-density matter, similar to what was discussed by Blake \& Schramm (1976).

On the other hand, Kuroda et al. (2008) started to explore for the first time systematically the consequences of the wind termination shock for the r-processing in the wind. Decelerating the outflow abruptly, the reverse shock does not only raise the entropy of the matter, but in particular it slows down the temperature and density decline that takes place in the subsequent expansion. Kuroda et al. (2008) pointed out that the change in the temperature behavior plays a decisive role in determining the r-process abundances, because the nucleosynthesis path depends strongly on the temperature during the r-process freezeout phase. In contrast, the entropy jump does not seem to be important because high entropies in the shocked outflows are reached only when the temperature is already well below $2.5 \times 10^{9} \mathrm{~K}$, i.e. not in the regime where the neutron-to-seed ratio is established before the onset of r-processing.

In the present paper we also investigate the influence of the late-time outflow dynamics on the r-process nucleosynthesis. To this end we consider outflow trajectories that consist of an initial homologous phase and a second, slower expansion stage. This describes the wind dynamics only schematically but at least some basic features that are found in detailed solutions of unshocked as well as shocked outflows can be reproduced. In contrast to the outflow solutions studied by Arnould et al. (2007), for example, our simple parametric ansatz for the temperature and density decrease in the ejected matter allows us to modify the early expansion behavior and that at late times independently, i.e. in an uncoupled way. This is similar to the approach taken by Wanajo et al. (2002) and closer to how, for example, the presence of a wind termination shock acts on the matter. Motivated by the insensitivity to the entropy jump seen by Kuroda et al. (2008), we ignore the discontinuity of the fluid variables at the location where their time dependence is assumed to change.

In order to obtain r-processing with reasonable instead of extreme values of the wind entropy $s$ and electron fraction $Y_{\mathrm{e}}$, we consider sufficiently short exponential timescales for the first expansion stage, i.e., we consider timescales in the ballpark of those given for example by Otsuki et al. (2000) for outflows from a neutron star with a gravitational mass of $2 M_{\odot}$, a radius of $10 \mathrm{~km}$, and a neutrino luminosity of $10^{52} \mathrm{erg} \mathrm{s}^{-1}$ (see their Table 1). We will vary the prescribed expansion behavior during the second phase in order to discuss the effects of the late-time temperature evolution on the r-processing from a more general point of view than done in previous works. The role of a strong or weak deceleration of the very fast, transsonic winds will be investigated by a constant or power-law behavior, respectively, of the temperature and density during the second stage. The former may be interpreted as the limiting case of a strong deceleration by a wind termination shock. The latter may be considered as an approximation to the reduced acceleration that a freely expanding wind experiences at larger radii after its roughly homologous initial phase.

Our paper is structured as follows. In Sect. 2 we will describe the dynamical model and the network used in our study, and will present some numerical tests we performed. In Sect. 3 we will describe our results and in Sect. 4 we finish with conclusions.

\section{Network, input data, and numerical modeling of the $(\alpha+r)$-process}

We consider here the conditions for r-process nucleosynthesis in neutrino-driven outflows from the surface layers of hot nascent neutron stars, which have been the subject of many previous studies, where different aspects of the problem were discussed (see, e.g. Qian \& Woosley 1996; Hoffman et al. 1997; Woosley et al. 1994; Cardall \& Fuller 1997; Qian \& Wasserburg 2000; Takahashi et al. 1994; Otsuki et al. 2000; Sumiyoshi et al. 2000; Thompson et al. 2001; Kuroda et al. 2008; Arnould et al. 2007, and references therein). Our main goal here is to evaluate numerically the possibility of producing r-process elements in this environment in dependence of the late-time behavior of the outflowing gas. Within the framework of a very simple, purely analytic model of the wind we want to determine the favorable combinations of entropy, $Y_{\mathrm{e}}$ and dynamical timescale, and how sensitively these parameters influence the outcome. Though, of course, our results will not allow us to make a judgement about whether neutrino-driven winds are the long-sought site of r-process material or not, our parametric approach has the advantage of reducing the dynamical aspects of the problem to an absolute minimum of ingredients, giving one much freedom in the choice of the involved parameter values.

\subsection{Outflow behavior}

We represent the outflow behavior during the early and late expansion phases by different analytic functions. These describe qualitatively (but certainly not to very high accuracy) on the one hand the wind acceleration through the sonic point, and on the other hand the evolution of the outflow during a second phase of either deceleration by a reverse shock or reduced acceleration.

In the first stage of the expansion of a spherical mass shell we assume a homologous velocity-radius dependence, $v \propto r$, corresponding to an exponential growth of the radius, $r(t)=$ $R_{\text {ini }} \exp \left(t / \tau_{\text {dyn }}\right)$. Therefore, steady-state conditions (which imply $r^{2} \rho v=$ const) yield an exponential decline of the density and for an adiabatically expanding, radiation-dominated wind (i.e., $\rho \propto T^{3}$ ) also an exponential decrease in the temperature:

$$
\begin{aligned}
\rho(t) & =\rho_{\text {ini }} \cdot \exp \left(-3 t / \tau_{\text {dyn }}\right), \\
T_{9}(t) & =T_{9}^{\text {ini }} \cdot \exp \left(-t / \tau_{\text {dyn }}\right) .
\end{aligned}
$$

Here $T_{9}$ is the temperature normalized to $10^{9} \mathrm{~K}$ and $\rho_{\text {ini }}$ and $T_{9}^{\text {ini }}$ are the initial values of density and temperature at some small radius $R_{\text {ini }}$. The dynamical timescale or expansion timescale $\tau_{\text {dyn }}$ will be treated as a free parameter and can vary between $1 \mathrm{~ms}$ and more than $100 \mathrm{~ms}$.

For reasons of simplicity, we will always assume that $\rho$ is proportional to $T^{3}$ and that the gas entropy per nucleon (in units of Boltzmann's constant $k_{\mathrm{B}}$ ) is given by the relation $s=3.34 T_{9}^{3} / \rho_{5}$ with $\rho_{5}=\rho /\left(10^{5} \mathrm{~g} \mathrm{~cm}^{-3}\right)$. One should, however, keep in mind that this is only a simplifying approximation, which is accurate only when the entropy of the gas is large 
enough ( $s \gtrsim 100 k_{\mathrm{B}}$ per nucleon) and the temperature sufficiently high $\left(T_{9} \gtrsim 5\right)$. In this case radiation-dominated conditions prevail, electrons are relativistic, electron-positron pairs are abundant, and baryons contribute to the total entropy only at the level of a few percent. In reality, however, the pairs begin to disappear below $T_{9} \approx 5$ and the ratio $T^{3} / \rho$ increases. Assuming it to be constant also at low temperatures therefore leads to an overestimation of the density in the outflowing matter compared to a truely adiabatic evolution (see Witti et al. 1994, for a detailed discussion).

The deceleration by the reverse shock or changing acceleration behavior are supposed to happen at a radius $r_{0}$ and time $t_{0}$ when the velocity, density, and temperature reach the values $v_{0}$, $\rho_{0}$, and $T_{0}$, respectively. As discussed in the introduction, we ignore here the discontinuous behavior of the dynamical and thermodynamical variables at the shock and connect the early-time behavior continuously with the late-time behavior. This means that also the ratio $T^{3} / \rho$ (and therefore the quantity $s$ that we consider as gas entropy) remains unchanged at the transition point and is taken to be constant during the following second stage of the expansion. For the latter we consider two cases with different limiting behavior for $t \gg t_{0}$. In the first case we assume that the density and temperature asymptote to constant values,

$\rho(t)=\rho_{0}$,

$T(t)=T_{0}$, for $t \geq t_{0}$.

For steady-state conditions this implies that the radius and the velocity of a Lagrangian mass shell evolve at $t \geq t_{0}$ according to

$r(t)=r_{0}\left[1+3 \frac{v_{0}}{r_{0}}\left(t-t_{0}\right)\right]^{1 / 3}$,
$v(t)=v_{0}\left[1+3 \frac{v_{0}}{r_{0}}\left(t-t_{0}\right)\right]^{-2 / 3}$,

and therefore $r(t) \propto t^{1 / 3}$ and $v(t) \propto t^{-2 / 3} \propto r^{-2} \rightarrow 0$ for $t \gg t_{0}$. We consider this choice of time-dependence as an approximative representation of the strong deceleration experienced by the wind in passing through a termination shock. Assuming constant density and temperature on the typical timescale of r-processing (several hundred milliseconds) is in reasonably good agreement with the slow evolution of the shocked outflow found in detailed hydrodynamic models (see Figs. 6 and 8 in Arcones et al. 2007).

In the second investigated case the density and temperature are still assumed to decline at late times, but much less steeply than during the exponential first expansion phase:

$$
\begin{aligned}
& \rho(t)=\rho_{0}\left(\frac{t}{t_{0}}\right)^{-2}, \\
& T(t)=T_{0}\left(\frac{t}{t_{0}}\right)^{-2 / 3}, \text { for } t \geq t_{0} .
\end{aligned}
$$

For steady-state conditions this corresponds to

$$
\begin{aligned}
& r(t)=r_{0}\left[1-\frac{v_{0} t_{0}}{r_{0}}+\frac{v_{0} t_{0}}{r_{0}}\left(\frac{t}{t_{0}}\right)^{3}\right]^{1 / 3}, \\
& v(t)=v_{0}\left[1-\frac{v_{0} t_{0}}{r_{0}}+\frac{v_{0} t_{0}}{r_{0}}\left(\frac{t}{t_{0}}\right)^{3}\right]^{-2 / 3}\left(\frac{t}{t_{0}}\right)^{2},
\end{aligned}
$$

which yields $r(t) \propto t$ and $v(t)=v_{0}^{1 / 3}\left(r_{0} / t_{0}\right)^{2 / 3}=$ const $>0$ for $t \gg$ $t_{0}$. A posteriori this asymptotic behavior justifies our choice of the power-law time-dependence for $\rho$ and $T$ in Eqs. (7) and (8).
Note that $v(t \rightarrow \infty)<v_{0}$, i.e. deceleration (and not just a slowdown of the acceleration) happens only at late times if $t_{0}>\tau_{\text {dyn }}$. Values of the parameters used for some of the considered model cases are listed in Table 1.

The slower decline of the density and temperature during the power-law phase may be considered as a simplified representation of a reduced wind acceleration occurring after an approximately homologous (i.e., $v \propto r$ ) early expansion. Neutrinodriven winds exhibit such a nearly homologous evolution and thus nearly exponential increase in the radius and velocity with time only up to some distance, but then the velocity continues to grow less rapidly even in the absence (or before) a possible strong deceleration by a termination shock. The basic properties of this behavior are captured by our approach.

\subsection{Calculating the nucleosynthesis}

At the onset of its expansion, the ejected matter in the neutrinodriven wind is very hot and composed of free neutrons and protons. With ongoing cooling the nuclear statistical equilibrium (NSE) shifts towards an increasing mass fraction of alpha particles until finally the recombination to heavy nuclei sets in. Provided the conditions of entropy, electron fraction, and expansion timescale are suitable, the production of elements between the first and second peaks of the abundance curve through charged particle reactions and neutron captures may occur. For sufficiently extreme conditions even third-peak elements and ( $A \sim 196)$ may be assembled. Typically starting our nucleosynthesis calculations at temperatures $T_{9} \sim 6$ and densities $\rho \sim 10^{5}-10^{6} \mathrm{~g} \mathrm{~cm}^{-3}$ (the initial density is chosen according to a specified value for the conserved gas entropy), the element formation in our model runs first proceeds mainly through chargedparticle reactions. As the temperature and density decrease, the importance of $(\alpha, \mathrm{X})$ - and inverse reactions diminishes and the nuclear flow begins to be driven by a dynamical r-process, provided a sufficient number of free neutrons is still present. The production of a small mass fraction of heavy nuclei with $Z \geq 26$ before the onset of rapid neutron captures significantly reduces the requirements for the neutron source (a smaller number of free neutrons is required).

The time-dependent concentrations of nuclear species, $Y(A, Z)$, during the r-processing as determined by reactions with neutrons, beta-decays, and fission processes are described by the nucleosynthesis network developed by Blinnikov \& Panov (1996) and Nadyozhin et al. (1998). This network was here extended by charged-particle reactions and a larger number of nuclear reactions and fission processes so that it is possible to handle both the initial $\alpha$-process and the subsequent r-process with the same code.

The number of nuclei and reaction equations included in calculations depends on boundary conditions and the employed nuclear mass model and can be as large as about 4300 . We considered the region of nuclei with $Z$ ranging from 3 to 100 . The minimum and maximum atomic mass values for each chemical element were determined by the proton and neutron drip lines.

The reaction rates entering the system of differential equations differ by tens of orders of magnitude. Thus, the system of equations for nuclear kinetics to be solved is a classical example of a stiff system of ordinary differential equations. We used one of the most effective methods to integrate such a stiff system of equations, Gear's method (Gear 1971). The description of the complete package of solver routines and its applications to the r-process calculations can be found in Nadyozhin et al. (1998). 
Table 1. Parameter values for some of our considered outflows and of relevance for third peak formation.

\begin{tabular}{rrrrrcccc}
\hline \hline Run & $\begin{array}{r}\tau_{\text {dyn }} \\
{[\mathrm{ms}]}\end{array}$ & $\begin{array}{r}\text { Entropy } \\
{\left[k_{\mathrm{B}} / \mathrm{N}\right]}\end{array}$ & $T_{9}^{\mathrm{f}}\left(t_{0}\right)$ & $\begin{array}{r}t_{0} \\
{[\mathrm{~ms}]}\end{array}$ & $\begin{array}{c}v_{\text {ini }} \\
{\left[\mathrm{km} \mathrm{s}^{-1}\right]}\end{array}$ & $\begin{array}{c}v_{0} \\
{\left[\mathrm{~km} \mathrm{~s}^{-1}\right]}\end{array}$ & $\langle A\rangle$ & $\left\langle Y\left(A_{3}\right) / Y\left(A_{2}\right)\right\rangle$ \\
\hline 1 & 5 & 105 & 1 & 9 & 2000 & 12000 & 118 & $10^{-10}$ \\
2 & 2.5 & 105 & 1 & 4.5 & 4000 & 24000 & 127 & 0.01 \\
3 & 5 & 145 & 1.4 & 8 & 2000 & 10000 & 128 & 0.075 \\
4 & 5 & 145 & 0.4 & 14 & 2000 & 33000 & 128 & 0.04 \\
5 & 2.5 & 145 & 1 & 4 & 4000 & 20000 & 144 & 0.45 \\
6 & 10 & 170 & 1 & 16 & 1000 & 5000 & 119 & $10^{-5}$ \\
7 & 5 & 170 & 1 & 8 & 2000 & 10000 & 145 & 0.75 \\
\hline
\end{tabular}

Nuclear mass values as predicted by FRDM (Möller et al. 1995) were used, the beta-decay rates were calculated in the framework of the QRPA-model (Kratz et al. 1993), and the reaction rates with neutrons were described according to the calculations of Cowan et al. (1991) and of Rauscher \& Thielemann (2000).

In the nucleosynthesis studies presented here, the triple $\alpha$ and $\alpha \alpha \mathrm{n}$ reactions of helium burning, $3 \alpha \rightarrow{ }^{12} \mathrm{C}$ and $\alpha+\alpha+\mathrm{n} \rightarrow{ }^{9} \mathrm{Be}$, respectively, along with their inverse reactions, were included. The rates for both processes were taken from the REACLIB library of Thielemann et al. (1987) and those for the reactions of heavier nuclei with protons and $\alpha$-particles from Rauscher \& Thielemann (2000).

\subsection{Code tests and comparisons}

Code tests for a number of different cases were performed by Panov et al. (2001a), and for a number of explored cases r-process calculations with the same rates gave practically the same isotopic yields as in the paper of Freiburghaus et al. (1999) Network calculations of the $\alpha$-process with the present code were compared with the results shown by Witti et al. (1994) and yield rather compatible abundance distributions at the beginning of the r-process, in spite of differences in the employed nuclear reaction rates and the nuclear mass model.

In this context we would like to note that the transition from the $\alpha$-process to the r-process can only be done correctly on the basis of the same mathematical model. In our calculations we use the same code for both parts of the nucleosynthetic reaction sequence, without artificial devision into $\alpha$ - and r-process steps.

We compared the seed production as calculated with our full network to results from nuclear statistical equilibrium (NSE) as obtained by Nadyozhin \& Yudin (2004), Blinnikov et al. (to be published), and with the NSE-code used by Witti et al. (1994). We observed rather good agreement at temperatures $T_{9} \approx 5-6$ and a density around $5 \times 10^{6} \mathrm{~g} \mathrm{~cm}^{-3}$. At such temperatures the $\alpha$ peak is already formed and the mass fraction of seed nuclei (i.e., heavy elements) is still small, the most abundant nuclei in both cases being ${ }^{50} \mathrm{Ti}$ and ${ }^{54} \mathrm{Cr}$. Small discrepancies between the different calculations emerge mostly from the use of different mass formulas and can lead to smaller differences in the subsequent $\alpha$ - and r-process. They may be important for exact predictions of the abundance flow during the r-processing, but they should not affect the basic results of our investigation.

We also compared our results for the $\alpha$-process with the calculations made by Witti et al. (1994), using conditions similar to those of the HT-model of Witti et al. (1993), i.e., $s=390$, $Y_{\mathrm{e}}=0.455$. For an exponential expansion with a timescale of $\tau_{\text {dyn }}=62 \mathrm{~ms}$, which is the same as in the HT-model, the number density $n_{\mathrm{n}}$ as a function of temperature (Fig. 1, left) is clearly different from what was obtained by Takahashi et al. (1994) (see Fig. 2 therein and the dashed line in the left panel of Fig. 1). When we increase the expansion timescale to $\tau_{\text {dyn }}=162 \mathrm{~ms}$, our result becomes close to that of Takahashi et al. (1994). The reason for this discrepancy is the fact that our calculations assume homologous expansion with an exponential decrease in density and temperature, whereas the temperature and density in the hydrodynamic model considered by Takahashi et al. asymptote to nonvanishing values, i.e., the temperature approaches $T_{9}=1.5$ during the later expansion. Although r-process elements are formed, neither of our exponential runs leads to the build-up of a strong third r-process peak, despite the high entropy. While in the case with the short expansion timescale $\left(\tau_{\text {dyn }}=62 \mathrm{~ms}\right)$ the rapid dilution of the matter prohibits the efficient formation of seed nuclei, the larger expansion timescale $\left(\tau_{\text {dyn }}=162 \mathrm{~ms}\right)$ leads to a strong $\alpha$-process with a lot of seed production and therefore a neutron-to-seed ratio that is too low for the generation of very heavy r-process nuclei. In contrast, Takahashi et al. (1994) obtained a prominant third abundance peak. In their calculation a sufficiently fast initial expansion prevents the formation of too much seed material before the r-process starts, and therefore the neutron-to-seed ratio remains high. The asymptoting temperature and density on the other hand support a high neutron number density for such a long time that neutron capture reactions can assemble nuclei also in the third peak.

For lower entropy, $s=145$, and higher neutron excess, $Y_{\mathrm{e}}=0.42$, (Fig. 1, right) the number of free neutrons can also remain large enough to allow for the onset of r-processing when the $\alpha$-process freezes out at a temperature of $T_{9} \sim 2$. In this case, however, much shorter dynamical timescales are needed. Figure 2 displays the decreasing seed formation and increasing neutron-to-seed ratio for smaller expansion timescales $\tau_{\text {dyn }}$.

How many neutrons per seed nucleus are needed to form the third abundance peak $(A \sim 196)$ ? Usually the number mentioned in this context is not less than 150. But this estimate is based on the simple calculation how many neutrons a single ${ }^{56} \mathrm{Fe}$ nucleus must capture to finally, after a chain of beta-decays, end as nucleus in the platinum peak. In the actual r-process, only a fraction of the nuclei that are initially formed by the alpha-process - we call these nuclei the "seed" - will be ultimately transformed to elements and isotopes with the highest mass numbers.

Taking into account that the observed ratio of the third to second abundance peak is $Y_{196} / Y_{130} \sim 0.2$, we estimate that the neutron number needed to obtain a strong third peak is of the order of $(196-60) \times 0.2 \approx 30$. The actual value might even be a bit lower, because the mean atomic number at the freeze-out time of the $\alpha$-process can be about 80 or more instead of 60 (an exact estimate, however, also depends on the conditions in which the r-processing takes place and the corresponding speed of the abundance flow). Therefore a solar-like formation of the 

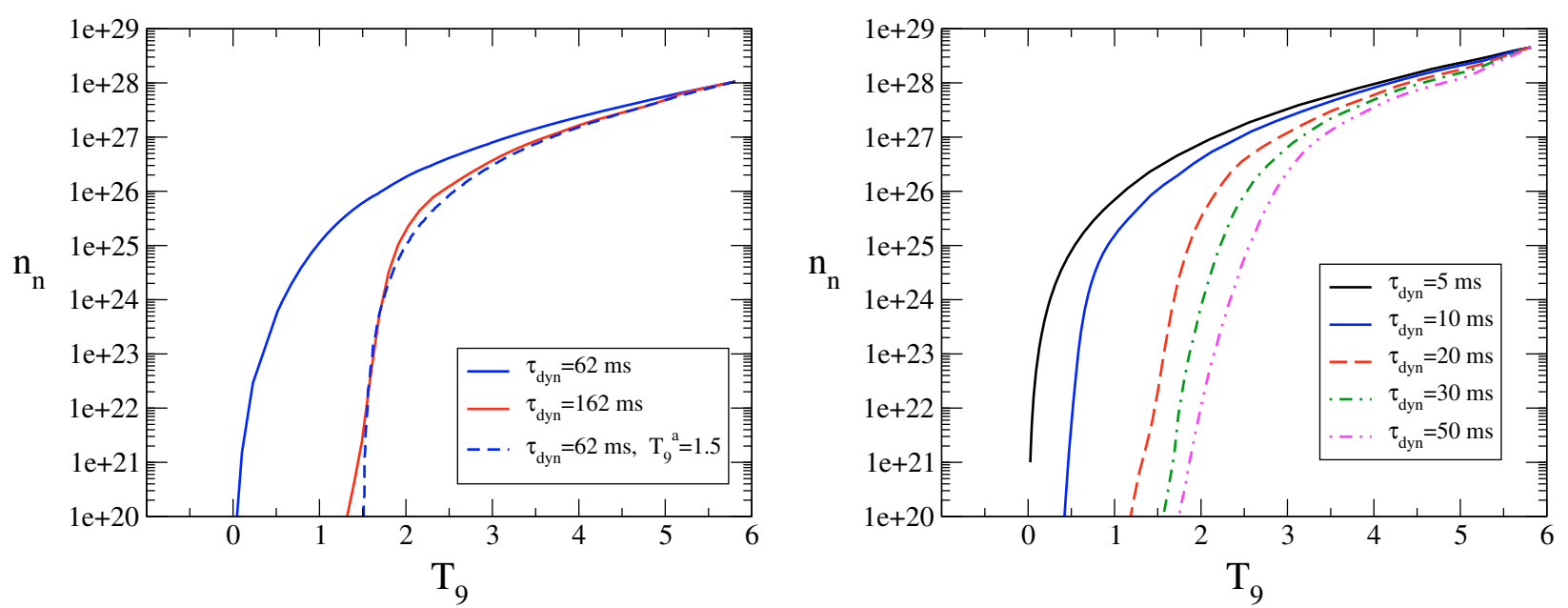

Fig. 1. $T_{9}$-dependence of the free neutron density $n_{\mathrm{n}}$ (in particles per $\mathrm{cm}^{-3}$ ) for $s=390$ (measured in $k_{\mathrm{B}}$ per nucleon), $Y_{\mathrm{e}}=0.46($ left $)$ and $s=145$, $Y_{\mathrm{e}}=0.42($ right $)$. The different curves correspond to different exponential expansion timescales $\tau_{\text {dyn }}$ as given in the list. The dashed line in the left plot shows the result for conditions close to those in Fig. 7 of Witti et al. (1994). In the case of the HT-model considered by Witti et al. (1994), the temperature asymptotes to a value of $T_{9}^{\mathrm{a}}=1.5$. The density also decreases so slowly that a high neutron number density is supported long enough for the creation of a strong third r-process peak. In contrast, during the free expansion of very high entropy material with an exponential timescale of $0.62 \mathrm{~ms}$, the matter dilutes too fast to produce large amounts of heavy elements, and no prominent third r-process abundance peak can be formed. In spite of a similar decline of the neutron density with temperature for an expansion timescale of $162 \mathrm{~ms}$, the third abundance peak can also not be assembled in this case, because the slow expansion leads to abundant seed production in a strong $\alpha$-process and too low a neutron-to-seed ratio.
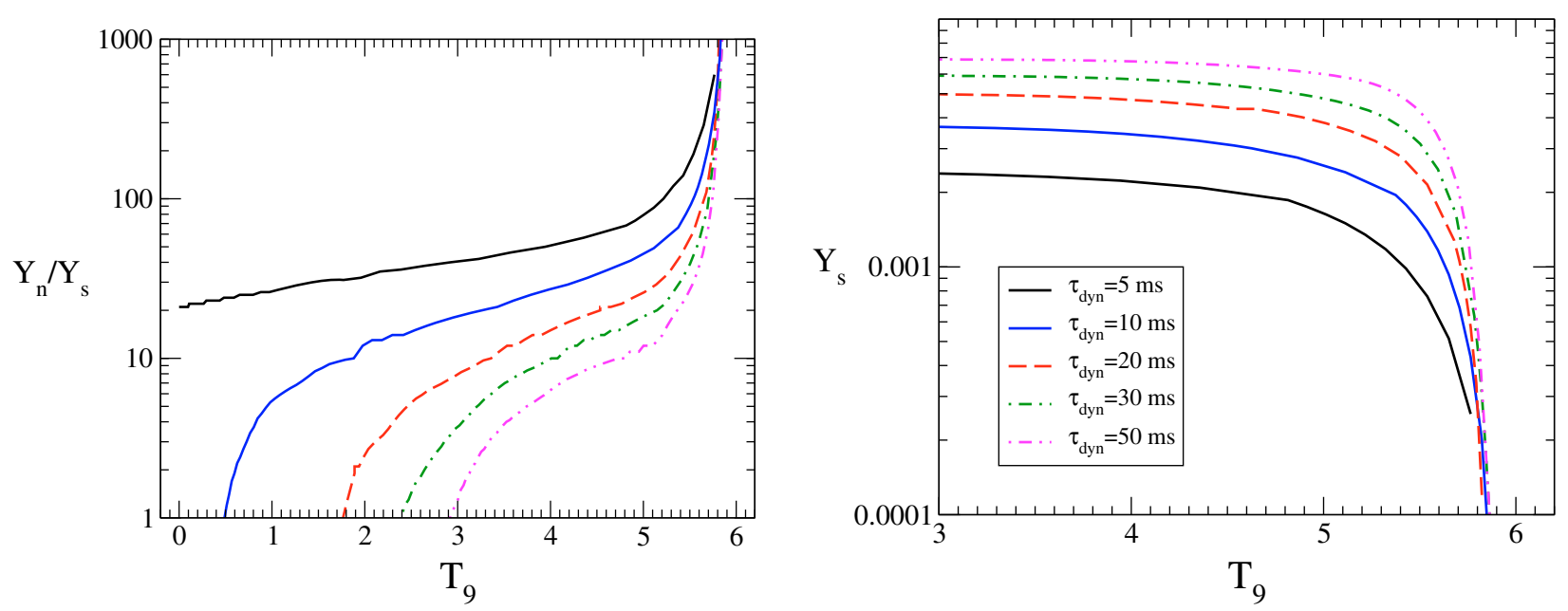

Fig. 2. $T_{9}$-dependence of the neutron-to-seed ratio $Y_{\mathrm{n}} / Y_{\mathrm{S}}($ left $)$ and of the "seed" number fraction $Y_{\mathrm{S}}($ right $)$ for an entropy of $s=145$ and $Y_{\mathrm{e}}=0.42$. The different curves correspond to different exponential expansion timescales, $\tau_{\mathrm{dyn}}=5,10,20,30,50 \mathrm{~ms}$ (the same cases as in the right panel of Fig. 1). At the beginning of r-processing $\left(T_{9} \approx 2\right)$, the free neutron number is sufficiently large to form heavy elements up to the second or even third peak if the dynamical time is less than $10 \mathrm{~ms}$ for the considered entropy and electron fraction. For higher values of $\tau_{\text {dyn }}$, the free neutrons are exhausted right at the beginning of the r-processing and the number of seed nuclei is too high.

platinum peak can be expected if one has a neutron-to-seed ratio of around 30 after the freeze-out of charged-particle reactions.

We point out, however, that this value does not ensure that the solar abundance distribution in the whole mass-number region between the second and third abundance peaks is reproduced well. This goal is not attempted in our study here, and it is well known that a superposition of at least two components with different physical conditions instead of a single ejecta trajectory (as considered by us) is needed for matching the solar r-process pattern (see, e.g. Kratz et al. 1993; Goriely \& Arnould 1996; Wanajo et al. 2004; Kuroda et al. 2008). In our fast-expansion cases we regard the described criterion as a reasonably good indicator for outflow conditions that allow for the appearance of a strong third peak during the r-processing that follows after the freeze-out of charged-particle reactions.

To judge about the possibility of an r-process for different choices of the parameters of our dynamical model $\left(s, Y_{\mathrm{e}}, \tau_{\mathrm{dyn}}\right)$, we first consider the seed formation and the corresponding time evolution of the neutron-to-seed ratio in the homologous expansion phase. In Fig. 1 we have seen agreement of the free neutron density as a function of time between our calculations and the prior ones by Takahashi et al. (1994) for a suitable choice of the expansion timescale $\tau_{\text {dyn }}$ (although the heavy-element nucleosynthesis was considerably different as discussed above). Figure 2 shows the neutron-to-seed ratios and the seed abundances, $Y_{\mathrm{s}}=\sum_{\mathrm{Z}>2} Y_{Z}$, versus temperature for the same cases 
as displayed in the right panel of Fig. 1. Note that we consider all nuclei with $Z>2$ as seeds, which is a different definition than used by Terasawa et al. (2001), who restriced seed nuclei to the more narrow range of $70 \leq A \leq 120$ and $Z>26$. The latter mass range is appropriate when the formation of the second abundance peak in an incomplete r-process is discussed. Here, however, we explore the possibility of third-peak production and consider a combined $\alpha$ - and r-process. In this case the atomic mass number range should not be constrained. Because of the very short expansion timescale and the treatment of both the $\alpha$-process and the r-process within the framework of the same mathematical model and computer code, a distinction of seed nuclei and r-processed material becomes artificial and one has to carefully judge when this information is measured and what it means for the evolution of the mass number with time. Often it refers to older calculations in which the $\alpha$ - and r-process were computed in two independent steps and with different codes. In this context we note that the amount of heavy nuclei in our calculations is approximately twice as big as found by Terasawa et al. (2002). This difference is mostly explained by the different model parameters (in particular, Terasawa et al. investigated outflows with higher entropies), but to some extent may also be a consequence of different nuclear rates and included reactions.

With the definition of seed nuclei adopted by us, the seed abundance becomes constant after the freeze-out of the alphaprocess and will also be unaffected by the transition to a second stage with modified expansion behavior for the cases considered by us. In contrast, replacing the free (homologous) expansion by a slower second stage of outflow behavior will have an impact on the density of free neutrons as a function of time. The free neutron density determines the r-process path and thus the formation of the heaviest elements in the third abundance peak.

From Fig. 2 (left panel) we see that the expansion timescale for third peak formation to happen should be less than about $10 \mathrm{~ms}$. In this case the neutron-to seed ratio $Y_{\mathrm{n}} / Y_{\mathrm{s}}$ as the decisive macroscopic factor for the platinum peak formation reaches the interesting values mentioned above. Naturally, this ratio depends not only on the number of free neutrons, but also on the seed abundance. Figure 2 (right panel) shows that the seed production varies strongly with the expansion timescale: if the dynamical time is large, a lot of seed is assembled and all neutrons will be captured faster than the platinum peak begins to appear. With shorter expansion timescale the seed production drops and at the same time the density of free neutrons increases (see right panel of Fig. 1). The consequence of both trends with reduced $\tau_{\text {dyn }}$ is a strong growth of the $Y_{\mathrm{n}} / Y_{\mathrm{s}}$ ratio that is present at the beginning of the r-process.

However, as already discussed above in the context of Fig. 1, too rapid expansion can also be disadvantageous for a strong r-process. When the dynamical timescale becomes very short, the expansion and gas dilution proceed faster than the rate of recombination of alpha particles to seed nuclei and subsequently the rate of neutron captures. For the conditions considered in Fig. 2 this happens when the homologous expansion and density decrease are too rapid to allow for the neutrons to be assembled into a strong 3rd peak, despite a high neutron-to-seed ratio and a sufficiently small seed concentration $Y_{\mathrm{S}}$ below a critical upper value of $Y_{\mathrm{s}}^{\mathrm{cr}} \sim 3 \times 10^{-3}$ after the freeze-out of charged-particle processes.

Of course, as discussed in detail in many previous works, besides the expansion timescale, the entropy and the neutron excess (or $Y_{\mathrm{e}}$ ), have a sensitive influence on the strength of the r-processing, i.e., on the question how many nuclei with mass numbers above the second abundance peak and in particular near the third peak can be formed. In any case, for rapid expansion ( $\operatorname{small} \tau_{\text {dyn }}$ ) the seed production is reduced and the neutron-toseed ratio becomes more favorable for a strong r-process. The heavier the seed nuclei at the end of the $\alpha$-process are, the lower can the required number of free neutrons be. This was already discussed by, e.g. Panov \& Chechetkin (2002), who showed that fairly low neutron/seed ratios are already sufficient when the seed material at the freeze-out time of charged-particle reactions consists mostly of nuclei in the second abundance peak.

\section{Asymptotic behavior of temperature and density and formation of the platinum peak}

Our combined $(\alpha+r)$-code was applied to nucleosynthesis calculations assuming the two-stage expansion behavior described above, with a second phase of either constant or slowly decreasing temperature and density following a first phase of rapid, exponential expansion. Our calculations were started at a temperature of $T_{9}=6$, assuming NSE at this point. For conditions similar to those given in Fig. 2 of Terasawa et al. (2001) with a short expansion timescale of $\tau_{\text {dyn }}=5 \mathrm{~ms}$, we obtained basically the same results for the development of the neutron number density and average neutron separation energy until about half a second, and found gross agreement of the structure of the abundance distribution. Smaller discrepancies might be attributed to differences in the initial composition and nuclear reaction rates. The number fraction of seed nuclei reported by Terasawa et al. (2001), $Y_{\mathrm{s}} \sim 0.001$, is a bit low compared to our results, but this is probably mainly caused by a different definition of "seeds".

In contrast, we were not able to confirm the formation of the third abundance peak for conditions similar to those considered by Terasawa et al. (2002) with an assumed expansion timescale of $\tau_{\text {dyn }}=25 \mathrm{~ms}$. Besides not providing exact information about the initial density and the kind of network used for the alpha- and r-process calculations (a full network or offline calculations for the r-processing?), some of the results and explanations are hard to reproduce in detail. For example, the calculations done by us with different NSE codes (including that of Nadyozhin \& Yudin 2004) and also the simulations by Witti et al. (1994) show that $\alpha$-particles reassemble mainly down to temperatures around $T_{9}=5-6$, somewhat dependent on the density, but not at temperatures as low as $T_{9}=4$ (see Fig. 1 in Terasawa et al. 2002). Moreover, they argued that a lower value of the asymptotic temperature is favorable for a successful r-processing by allowing for a higher neutron-to-seed ratio, because such a lower "outer boundary" temperature reduces charged-particle reactions and thus the production of seed nuclei. This suggested influence of the chosen asymptotic temperatures in the range between $T_{9}=0.4$ and $T_{9}=1.3$, however, is implausible, because charged-particle reactions become inefficient already at temperatures of $T_{9} \approx 2$, when the thermal energies of protons and $\alpha$-particles become too low for enabling these particles to overcome the nuclear Coulomb barriers.

We will therefore not further attempt to compare our calculations with those done by Terasawa et al. (2002). Instead, we will in the following present our results for the formation of the third abundance peak in dependence of entropy, electron fraction, and dynamical timescale during the first, exponential expansion phase. In particular we will also study variations with the asymptotic values of temperature and density during the second, slower phase of expansion. This will help us in the analysis of the physical effects that can explain our results. Our goal is to develop a deeper understanding of the influence of the late-time 


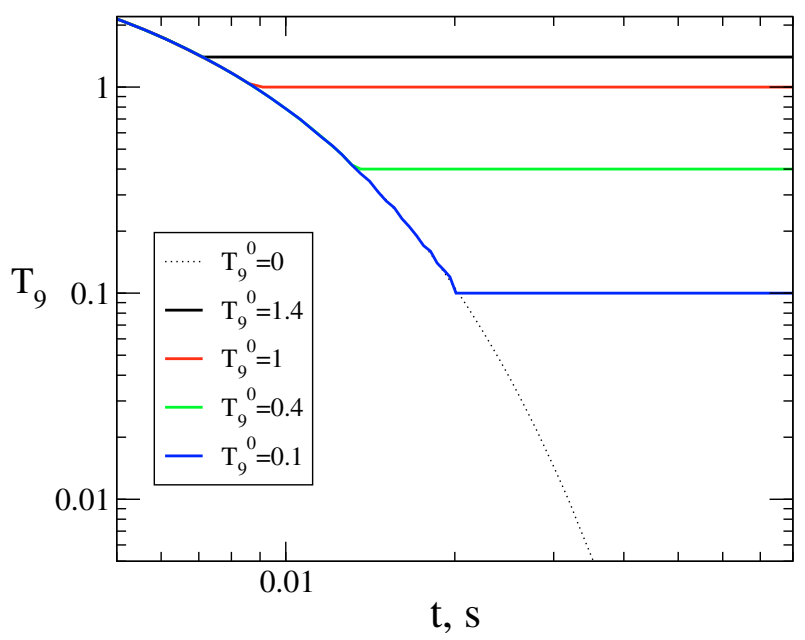

Fig. 3. Time evolution of the temperature for the different considered cases with exponential first expansion phase (starting at $T_{9} \sim 6$ and declining with a dynamical timescale of $\tau_{\mathrm{dyn}}=5 \mathrm{~ms}$ ) and constant later phase.

behavior of neutrino-driven winds on the possibility of strong r-processing in such an environment.

To this end we carried out a set of calculations for exponential timescales in the range from 1.0 to $25 \mathrm{~ms}$ and for four values of the wind entropy (in units of Boltzmann's constant per nucleon), $s=105,145,170$, and 200 . The initial neutron to proton ratio was determined by an electron fraction of usually $Y_{\mathrm{e}}=0.42$; some runs were performed with a value of $Y_{\mathrm{e}}=0.46$.

Our special attention was on the second stage of slower expansion, which was chosen to either proceed with constant temperature and density (Eqs. (3)-(6)) or gradually decreasing temperature and density (Eqs. (7)-(10)). In the former case we defined the asymptotic temperature as $T_{9}^{\mathrm{f}}\left(t \geq t_{0}\right) \equiv T_{9}^{0}=\mathrm{const}$ (Sect. 3.1), in the latter case the temperature was assumed to follow a power-law time-dependence according to $T_{9}^{\mathrm{f}}(t) \equiv T_{9}^{\mathrm{f}}\left(t_{0}\right) \times$ $\left(t_{0} / t\right)^{2 / 3}$ for $t \geq t_{0}$ (Sect. 3.2). The range of temperatures $T_{9}^{\mathrm{f}}\left(t_{0}\right)$, where the second expansion phase began, had a broad overlap with the "boundary temperatures" considered by Terasawa et al. (2001, 2002) and Wanajo et al. (2002).

Our standard calculations were performed with an entropy of $s=145$. Values of less than $s \approx 100$ turned out to lead only to the production of the second abundance peak around $A \sim 130$, but r-processing up to the platinum peak was not possible when the other characteristic wind parameters were varied within the limits mentioned above.

\subsection{Constant asymptotic temperature and density}

In this section we consider an exponential first expansion phase that is superseded at evolution time $t_{0}$ by a second, slow expansion phase whose asymptotic velocity at large radii goes to zero. In this case the density and temperature during the second phase adopt constant values (Eqs. (3)-(6)). We explore various choices of the asymptotic temperature $T_{9}^{\mathrm{f}}\left(t \geq t_{0}\right) \equiv T_{9}^{0}=\mathrm{const}$ between 0.1 and 1.4 (see Fig. 3). For an entropy of $s=145$, for which results are displayed in Figs. 4-6, these temperatures correspond to constant densities $\rho_{0}$ between about $1 \mathrm{~g} \mathrm{~cm}^{-3}$ and $10^{4} \mathrm{~g} \mathrm{~cm}^{-3}$. For these values the neutron number density at time $t_{0}$ shows differences by more than three orders of magnitude (see Fig. 4). Although the lowest assumed value of $T_{9}=0.1$ might appear extreme, because it requires very rapid exponential expansion for a longer time with fairly high velocities at the end of this phase (see Table 1), the wide range of asymptotic temperatures allows us to better understand the dependence of the nucleosynthesis on the late-time expansion behavior of the outflowing matter.

When the asymptotic temperature is as high as $T_{9}^{0} \geq 1.4$, the third peak hardly develops (see the abundance distribution at the end of our calculations in the right panel of Fig. 4). Only when the asymptotic temperature is reduced from this value to smaller numbers, the platinum peak grows in strength, because the free neutrons are exhausted more rapidly and the r-processing proceeds faster towards the high mass-number region. The latter fact can be seen by comparing the decrease in the neutron density with time for $T_{9}^{0}=1.4,1$, and 0.4 in the left panel of Fig. 4, and it is also visible from the neutron-to-seed ratios $Y_{\mathrm{n}} / Y_{\mathrm{s}}$ as functions of time in the left panel of Fig. 5. When the asymptotic temperature is lowered to less than $T_{9}^{0} \approx 0.3$, the free neutron density $n_{\mathrm{n}}$ during the slow-expansion phase becomes so low that the formation of the third abundance peak under such conditions slows down considerably. Correspondingly, $n_{\mathrm{n}}$ as well as $Y_{\mathrm{n}} / Y_{\mathrm{s}}$ decrease less quickly (see again the left panels of Figs. 4 and 5) and the height of the third peak at the time of neutron exhaustion becomes clearly lower (Fig. 4, right panel).

This inversion of the third peak formation with decreasing value of the asymptotic temperature $T_{9}^{0}$ can be clearly seen from the time evolution of the average of the yields in the platinum peak relative to those in the $N=82$ peak, $\left\langle Y\left(A_{3}\right) / Y\left(A_{2}\right)\right\rangle$, in the right panel of Fig. 5. The displayed quantity is defined by summing up the produced yields for five mass numbers around $A=130$ and $A=196$ and then computing the ratio ${ }^{2}$

$$
\left\langle\frac{Y\left(A_{3}\right)}{Y\left(A_{2}\right)}\right\rangle \equiv \frac{\sum_{193}^{197} Y\left(A_{i}\right)}{\sum_{128}^{132} Y\left(A_{i}\right)} .
$$

The left panel of Fig. 6 shows the sensitivity of the final value of this ratio to the chosen temperature $T_{9}^{\mathrm{f}}\left(t>t_{0}\right)=T_{9}^{0}$. While the growing strength of the platinum peak with lower asymptotic temperature agrees with the finding by Terasawa et al. (2002), their conclusion of a more efficient r-processing for lower "outer boundary pressure/temperature" obviously does not hold any more when this temperature is lower than $T_{9} \approx 0.3$, which is below the range explored by them.

We note here that often the change in the average atomic number is used for following the assembling of heavy elements beyond the $A \approx 130$ abundance peak. We found this quantity to be less suitable for this purpose than our height ratio $\left\langle Y\left(A_{3}\right) / Y\left(A_{2}\right)\right\rangle$, in particular when the second peak is much stronger than the third. A growth of the latter by one order of magnitude can mean a change in $\langle A\rangle$ by just one or a few units, whereas $\left\langle Y\left(A_{3}\right) / Y\left(A_{2}\right)\right\rangle$ varies sensitively and thus serves well as a tracer of changes in the abundance distribution in the highmass number region. The numbers listed in Table 1, for example, confirm the usefulness, in fact the superiority, of the ratio $\left\langle Y\left(A_{3}\right) / Y\left(A_{2}\right)\right\rangle$ instead of the mean mass number, $\langle A\rangle$, as an indicator of the third peak formation at least in the cases with very fast outflow expansion considered here (of course, again we do not claim that a single parameter value or a single outflow trajectory is sufficient for getting a good match of the whole r-process abundance distribution between the second and third peaks). While $\left\langle Y\left(A_{3}\right) / Y\left(A_{2}\right)\right\rangle$ exhibits a rapid growth during the build-up of the third abundance peak and changes by many orders of magnitude between cases with weak or strong third peak,

\footnotetext{
2 A similar peak ratio was considered in a recent paper by Beun et al. (2008).
} 

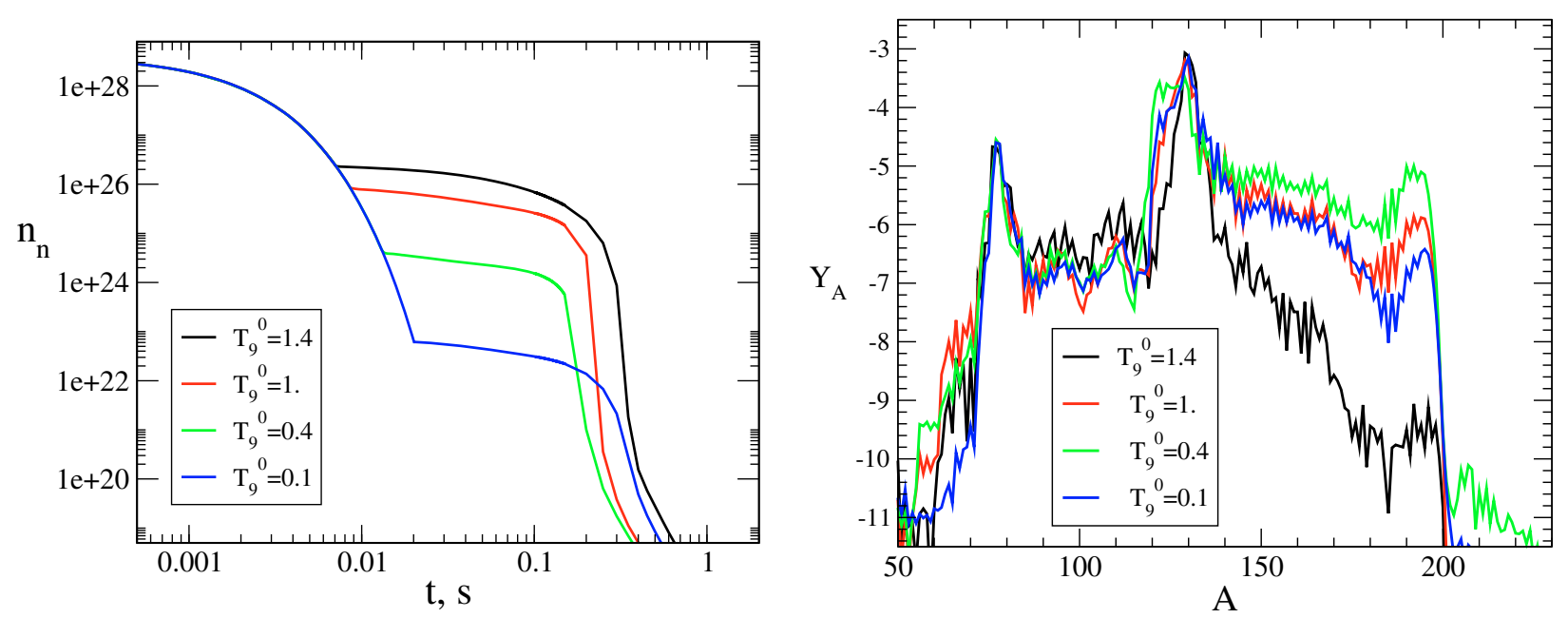

Fig. 4. Time dependence of the neutron number density, $n_{\mathrm{n}}(t)$ (left, in particles per $\left.\mathrm{cm}^{-3}\right)$, and the final abundance distributions, $Y_{\mathrm{A}}$, resulting from our network calculations for $s=145, Y_{\mathrm{e}}=0.42$, a short dynamical timescale of $\tau_{\text {dyn }}=5 \mathrm{~ms}$, and different values of the constant asymptotic temperature $T_{9}^{\mathrm{f}}\left(t \geq t_{0}\right)=T_{9}^{0}\left(t_{0}\right)$ during the second stage of the ejecta expansion. The corresponding values of $T_{9}^{0}$ are listed in the inset and the temperature evolution of function of time is shown in Fig. 3.
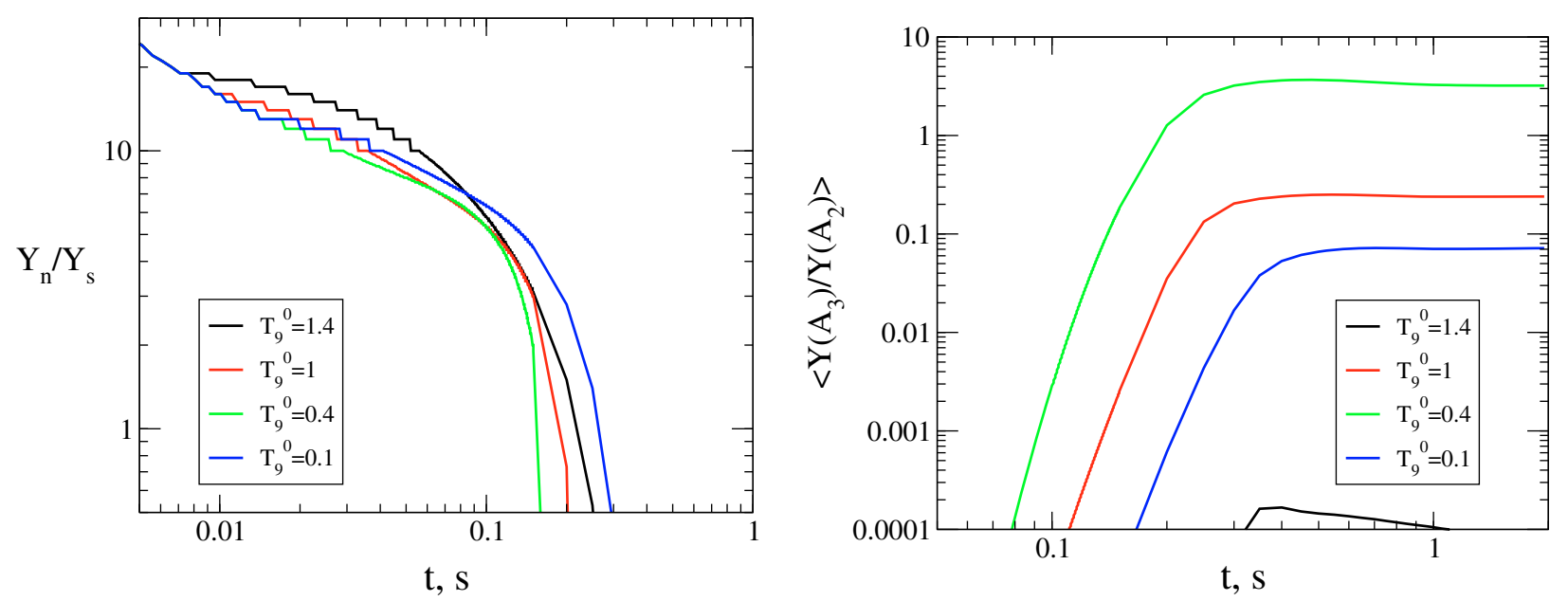

Fig. 5. Time dependence of the neutron-to-seed ratios, $Y_{\mathrm{n}} / Y_{\mathrm{S}}$ (left), and of the height of the third abundance peak relative to the second, $\left\langle Y\left(A_{3}\right) / Y\left(A_{2}\right)\right\rangle$ (right, in percent) for the same conditions as in Fig. $4\left(s=145, Y_{\mathrm{e}}=0.42, \tau_{\text {dyn }}=5 \mathrm{~ms}, T_{9}^{0}=\right.$ const. as given in the inset). The inversion of the third peak formation with decreasing asymptotic temperature can be clearly seen: when $T_{9}^{0}$ is lowered from 1.4 to 0.4 , the ratio of the third peak to the second increases, and when $T_{9}^{0}$ is reduced even further, $\left\langle Y\left(A_{3}\right) / Y\left(A_{2}\right)\right\rangle$ begins to drop again.

the average mass number remains within the relatively narrow interval of $118 \leq\langle A\rangle \leq 145$ (see Table 1).

The variation of the strength of the third peak with different asymptotic temperatures can be understood from the sensitivity of the neutron capture rates and nuclear photodisintegration rates to the neutron number density and temperature, respectively, and by the competition of these rates. This competition determines the location of the r-process path and thus the speed of the nucleosynthesis, which is defined by the $\beta$-decay rates. When the temperature during the second, slow expansion phase is large $\left(T_{9}^{0} \gtrsim 1.0\right)$, the $(\gamma, \mathrm{n})$-reactions are very fast and the r-process path lies closer to the stability region than in case of smaller $T_{9}^{0}$. The r-process flow beyond the second peak is then rather weak because of the low beta-decay rates and a correspondingly slow progression of the nuclear flow. When the asymptotic temperature is reduced to $T_{9}^{0} \approx 0.2-0.7$, the $(\gamma, \mathrm{n})$-rates decrease and the r-process path moves towards the neutron-drip line where the $\beta$-decay rates are higher. Therefore the r-processing proceeds faster beyond the second peak, leading to a more rapid drop of the free neutron density and a more efficient third peak production. When the asymptotic temperature is lowered to less than $T_{9}^{0} \approx 0.2$ during the late expansion phase, the neutron densities are very low so that the r-process path returns to a location closer to the $\beta$-stable region. In this case the $\beta$-decays again become slower and therefore the r-process nucleosynthesis decelerates and the third peak builds up to a smaller height.

These movements of the r-process path are a consequence of the different influence of a change in the asymptotic temperature on the $(\gamma, \mathrm{n})$ - and the neutron captures rates. For asymptotic temperatures in the interval $0.2 \lesssim T_{9}^{0} \lesssim 1.0$ the $(\gamma, \mathrm{n})$-rates are very sensitive to temperature variations. In contrast, the neutron capture rates change with the corresponding variations of the density and free neutron density less strongly. A reduced temperature therefore decreases the photodisintegration rates significantly, whereas the neutron captures remain fast despite the lower neutron density. When $T_{9}^{0} \lesssim 0.2$, the $(\gamma, \mathrm{n})$-rates become 

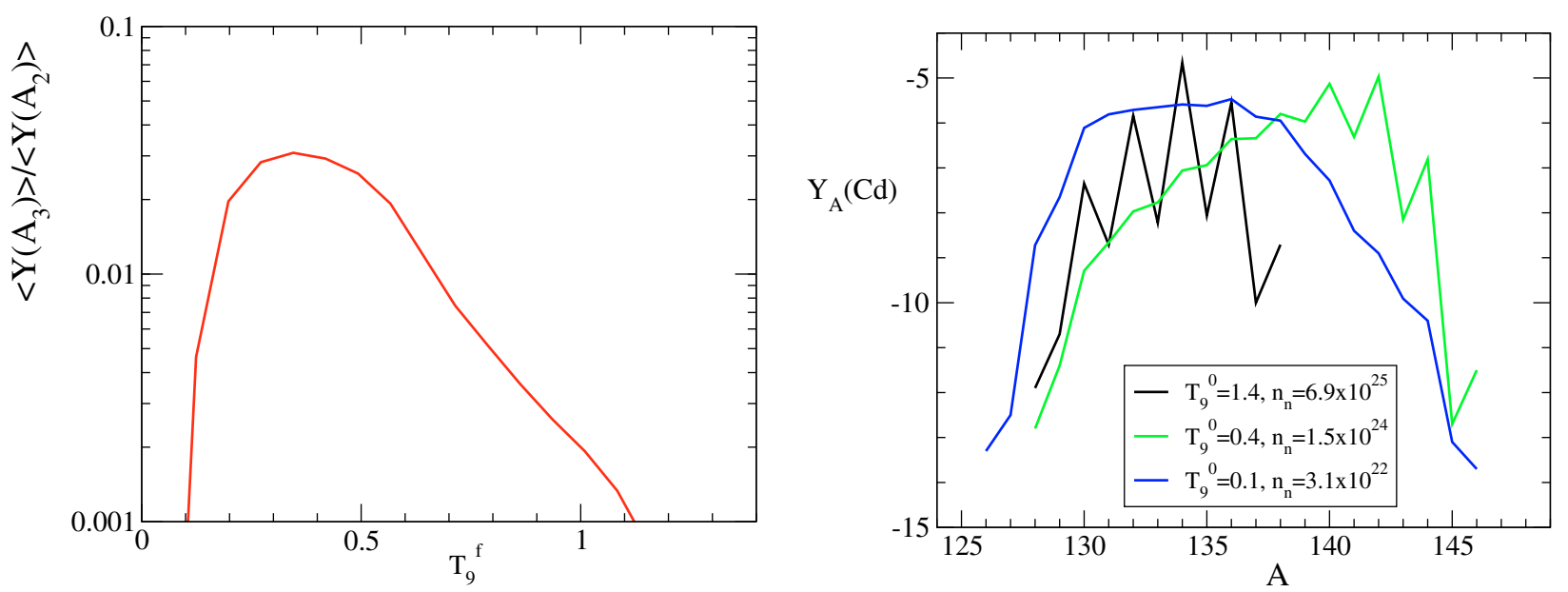

Fig. 6. Left: the changing strength of the third abundance peak, measured in terms of the ratio $\left\langle Y\left(A_{3}\right) / Y\left(A_{2}\right)\right\rangle$, as function of the asymptotic temperature $T_{9}^{\mathrm{f}}\left(t>t_{0}\right)=T_{9}^{0}$ for the conditions considered in Fig. $4\left(s=145, Y_{\mathrm{e}}=0.42, \tau_{\mathrm{dyn}}=5 \mathrm{~ms}, T_{9}^{0}=\right.$ const. $)$. The relative height of the third abundance peak depends strongly on the value of $T_{9}^{0}$ because of a sensitive influence of this temperature on the r-process path. This can be seen in the distribution of cadmium isotopes at an evolution time of $t=0.1 \mathrm{~s}$ for three different cases of the asymptotic temperature (right; the neutron number densities in the inset are those at $t=0.1 \mathrm{~s}$ in Fig. 4). When $T_{9}^{0}$ is lowered from 1.4 to 0.4 , the free neutron density and thus the neutron capture rate in the slow, second phase of the expansion decreases less strongly than the $(\gamma, \mathrm{n})$-rates, which are extremely sensitive to the temperature. As a consequence, the r-process path is shifted towards the neutron-drip line (compare the isotope distribution given by the green line) where the $\beta$-decay rates are higher and the r-processing proceeds so rapidly that there is time to form a strong third abundance peak. For even lower asymptotic temperature the $(\gamma, \mathrm{n})$-rates do not play an important role and the strength of the r-processing is determined by the free neutron density and the $\beta$-decay rates. Since the r-process path returns again to a location closer to the valley of stability (see the isotope distribution of the blue line), the $\beta$-decay rates are lower and the nucleosynthesis slows down. Therefore a strong third peak cannot be formed.

less relevant, but neutron captures still compete with $\beta$-decays. For such low temperatures and thus low neutron densities, the neutron capture rates are too low to drive the r-process path far away from the valley of stability.

The shift of the r-process path that is caused by different asymptotic temperatures (and connected parameters) manifests itself in different isotopic profiles of the elements formed by the r-processing (i.e. in different yield of the isotopes of an element). This can be seen for the case of cadmium in the right panel of Fig. 6.

\subsection{Power-law time-dependence of the asymptotic temperature and density}

In this section we consider the case that the initial exponential phase is superseded at time $t=t_{0}$ by a slow expansion phase in which the temperature and density decay according to power laws, i.e., $T_{9}^{\mathrm{f}}(t) \propto T_{9}^{\mathrm{f}}\left(t_{0}\right) / t^{2 / 3}$ and $\rho^{\mathrm{f}}(t) \propto \rho^{\mathrm{f}}\left(t_{0}\right) / t^{2}$ for $t \geq t_{0}$ (Eqs. (7)-(10)). The temperature evolution as function of time for the considered cases is displayed in Fig. 7.

For our standard set of wind parameters, $s=145$ (in units of Boltzmann's constant per nucleon), $Y_{\mathrm{e}}=0.42$, and $\tau_{\text {dyn }}=5 \mathrm{~ms}$ already used in Sect. 3.1, Fig. 8 shows the time evolution of the free neutron density (left panel) and the final abundance distributions for four different values of the transition temperature $T_{9}^{\mathrm{f}}\left(t_{0}\right)$ (right panel). The formation of the third abundance peak turns out to be fairly insensitive to variations of $T_{9}^{\mathrm{f}}\left(t_{0}\right)$ between about 0.4 and 1.4. For all transition temperatures in this interval the temperature evolution is very similar (Fig. 7) and a prominent third peak appears.

Since the temperature in the post-exponential phase drops rapidly, the strongly temperature-dependent $(\gamma, n)$-reactions become unimportant very soon, while the considered entropy allows a high free neutron density $\left(n_{\mathrm{n}} \geq 10^{22} \mathrm{~cm}^{-3}\right)$ to be present still for a long time. At such conditions the r-process path moves

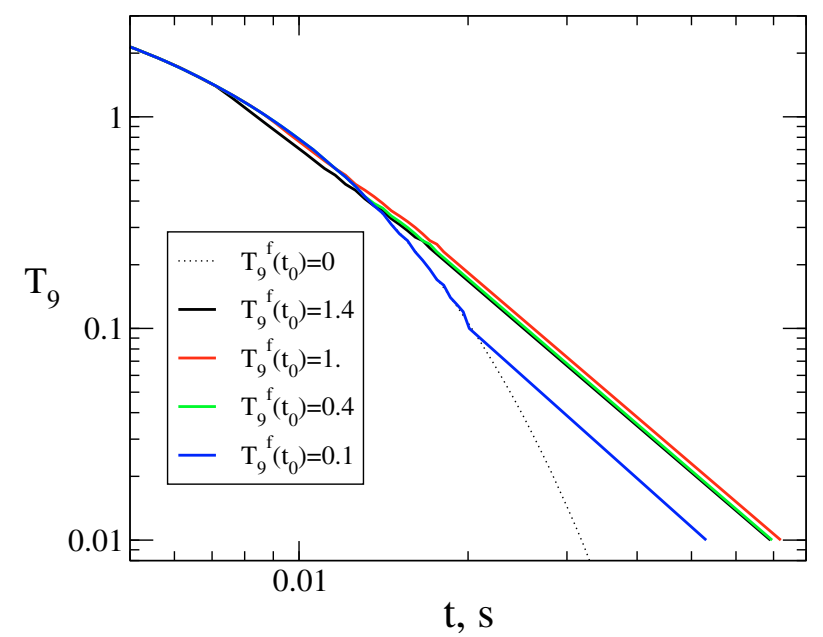

Fig. 7. Time evolution of the temperature for the different considered cases with exponential first expansion phase (starting at $T_{9} \sim 6$ and declining with a dynamical timescale of $\tau_{\text {dyn }}=5 \mathrm{~ms}$ ) and power-law later phase.

very close to the neutron-drip line and returns to the classical r-process path during free neutron exhaustion (Panov 2003). For the considered conditions its location shifts significantly only when the free neutron density changes by $2-3$ orders of magnitude. This explains the relative robustness of the abundance yields to variations of $T_{9}^{\mathrm{f}}\left(t_{0}\right)$ around 1.0.

The left panel of Fig. 9 displays the evolution of the neutronto-seed ratios $Y_{\mathrm{n}} / Y_{\text {seed }}$ that correspond to the neutron number densities of Fig. 8. With an entropy of $s=145$ and an exponential timescale $\tau_{\text {dyn }}=5 \mathrm{~ms}$ the values of $Y_{\mathrm{n}} / Y_{\text {seed }}$ at the time when the r-process has made the second abundance peak (at $t \approx t_{0}$ ) are around 20 for $T_{9}^{\mathrm{f}}\left(t_{0}\right)=0.4-1.4$. As discussed in Sect. 2, this is a slightly insufficient number of neutrons per seed 

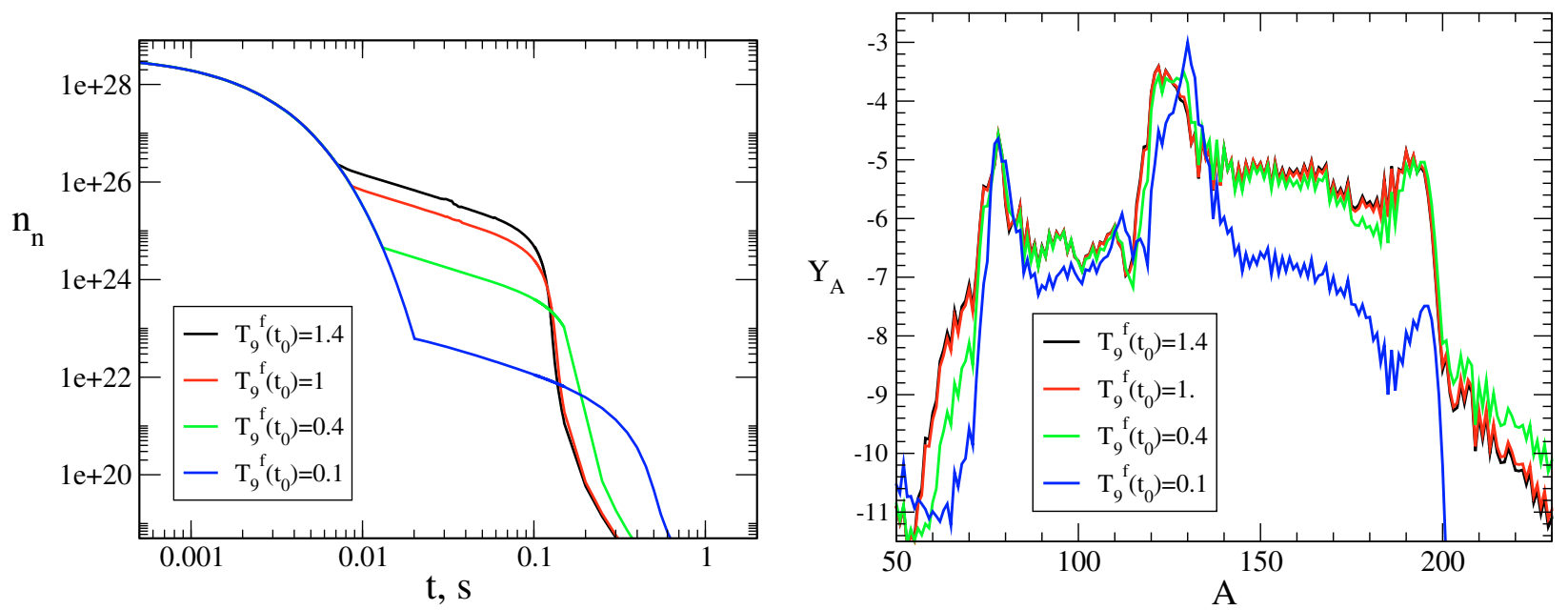

Fig. 8. Same as Fig. 4 (with $s=145, Y_{\mathrm{e}}=0.42$, and an exponential timescale $\tau_{\text {dyn }}=5 \mathrm{~ms}$ ), but for a power-law decay of the temperature during the second, slow expansion phase: $T_{9}^{\mathrm{f}}(t) \propto T_{9}^{\mathrm{f}}\left(t_{0}\right) / t^{2 / 3}$ for $t \geq t_{0}$ (see Fig. 7). The left plot shows the time dependence of the neutron number density, $n_{\mathrm{n}}(t)$ (in particles per $\mathrm{cm}^{-3}$ ), the right plot the corresponding final abundance distribution for different values of the transition temperature $T_{9}^{\mathrm{f}}\left(t_{0}\right)$ as given in the inset. Different from the case with constant asymptotic temperature in Fig. 4, the temperature and thus the photodisintegration rates drop rapidly also in the slow expansion phase. Neutron captures are therefore able to produce a prominent third abundance peak even for the highest considered value of the transition temperature, $T_{9}^{\mathrm{f}}\left(t_{0}\right)=1$.4. The height of the third peak relative to the second is very similar in the whole range of temperatures $T_{9}^{\mathrm{f}}\left(t_{0}\right)$ between 0.4 and 1.4 , and the inversion behavior of $\left\langle Y\left(A_{3}\right) / Y\left(A_{2}\right)\right\rangle$ with $T_{9}^{\mathrm{f}}\left(t_{0}\right)$ is absent.
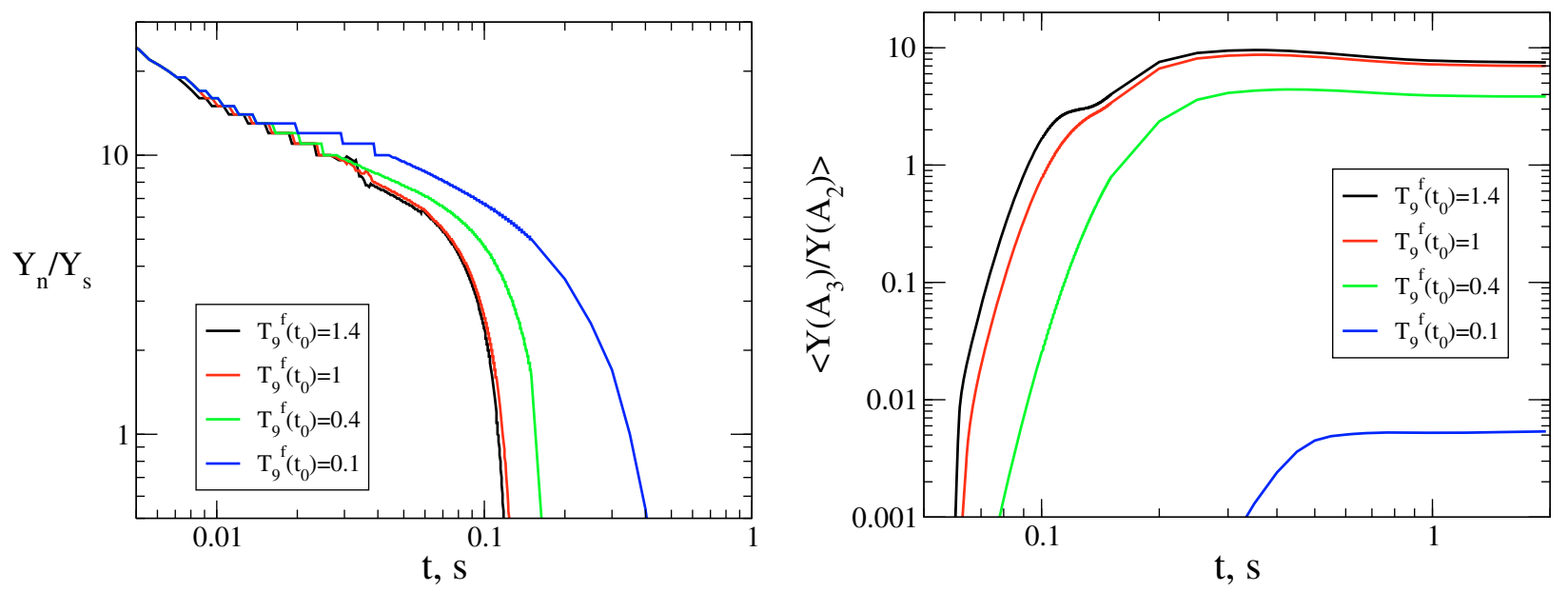

Fig. 9. Same as Fig. 5 (with $s=145, Y_{\mathrm{e}}=0.42$, and an exponential timescale $\tau_{\text {dyn }}=5 \mathrm{~ms}$ ), but for a power-law decay of the temperature during the second, slow expansion phase: $T_{9}^{\mathrm{f}}(t) \propto T_{9}^{\mathrm{f}}\left(t_{0}\right) / t^{2 / 3}$ for $t \geq t_{0}$ (as considered in Fig. 8). The left plot shows the time-dependent neutron-to-seed ratio $Y_{\mathrm{n}} / Y_{\mathrm{s}}$, the right plot the height of the third abundance peak relative to the second $\left(\left\langle Y\left(A_{3}\right) / Y\left(A_{2}\right)\right\rangle\right.$, in percent) as functions of time. The weak dependence of the r-processing on variations of the transition temperature $T_{9}^{\mathrm{f}}\left(t_{0}\right)$ between 0.4 and 1.4 as visible in the right panel of Fig. 8 is also evident from these two plots.

nucleus to create a third peak with exactly the observed yields. The small underabundance of the third peak can also be seen in the right panel of Fig. 9, where the height of the platinum peak relative to the tellurium peak is given as function of time. Different from the case of constant asymptotic temperature (see Fig. 5), $\left\langle Y\left(A_{3}\right) / Y\left(A_{2}\right)\right\rangle$ is nearly the same for $T_{9}^{\mathrm{f}}\left(t_{0}\right)=1.0$ and 1.4 , and is only slightly reduced for $T_{9}^{\mathrm{f}}\left(t_{0}\right)=0.4$. For even lower transition temperatures the height of the third peak drops steeply. This is evident from the blue line in the left panel of Fig. 10 and is a consequence of a faster decrease in the free neutron density (compare the left panels in Figs. 4 and 8), which for $T_{9}^{\mathrm{f}}\left(t_{0}\right)<0.4$ falls quickly to a value of $n_{\mathrm{n}} \sim 10^{22} \mathrm{~cm}^{-3}$, below which the equilibrium of $\mathrm{n}$-captures and $\beta$-decays is shifted to a region of slow beta-decay rates, leading to reduction of the third peak.
The left panel of Fig. 10 provides a direct comparison of the variation of $\left\langle Y\left(A_{3}\right) / Y\left(A_{2}\right)\right\rangle$ with the transition temperature between the exponential first expansion phase to the second phase of either constant temperature or power-law temperature decrease. Above a transition temperature $T_{9}^{\mathrm{f}}\left(t_{0}\right) \sim 0.4$ the behavior in both cases is dramatically different. While in the case of constant asymptotic temperature the relative height of the third peak decreases with higher values of $T_{9}^{0}$ (see Fig. 6 and Sect. 3.1), a power-law decay of the temperature in the second expansion phase leads to a prominent platinum peak for all values of $T_{9}^{\mathrm{f}}\left(t_{0}\right)$ between 0.5 and 1.4. The reason is again the large sensitivity of the photodisintegration reactions to the late-time behavior of the temperature. Because of the power-law decline the temperature drops within milliseconds to values where a $(n, \gamma)-(\gamma, n)$ equilibrium is no longer possible but is replaced by a quasi-equilibrium 

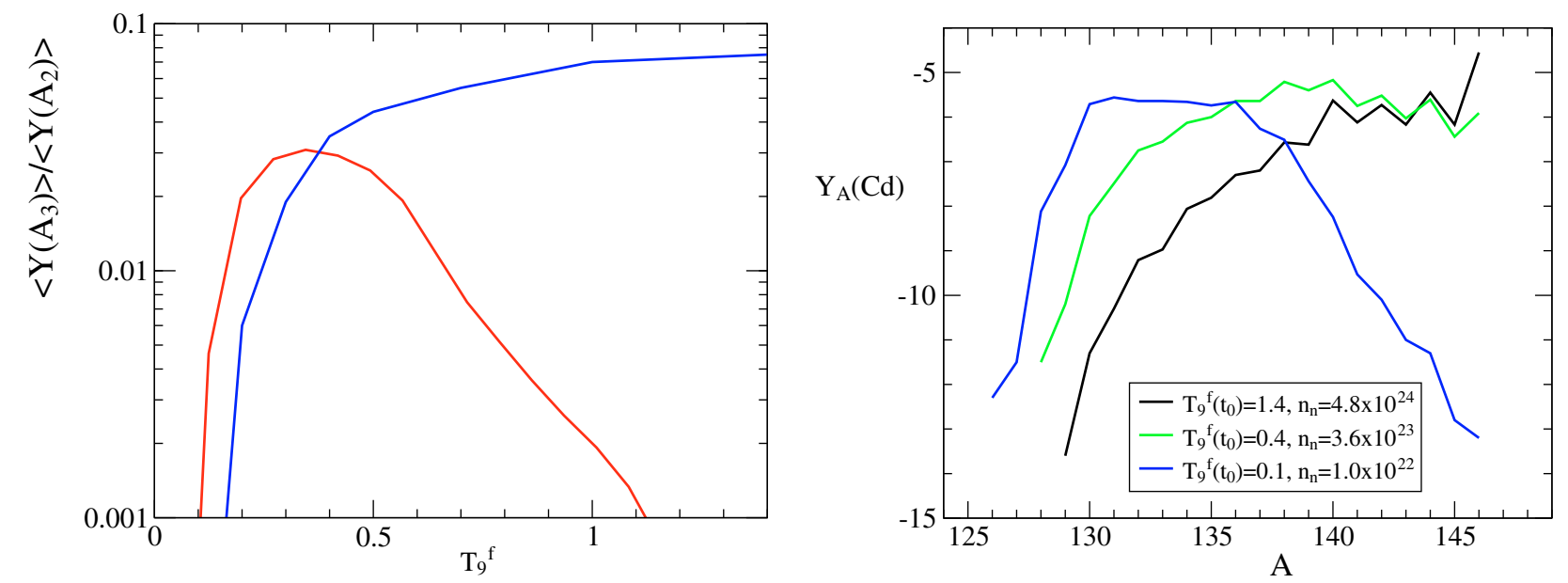

Fig. 10. Left: height of the third abundance peak relative to the second, measured in terms of the ratio $\left\langle Y\left(A_{3}\right) / Y\left(A_{2}\right)\right\rangle$, as function of the temperature $T_{9}^{\mathrm{f}}\left(t_{0}\right)$ for a power-law decay of the temperature during the second, slow expansion phase (blue curve) compared to the dependence of this quantity on $T_{9}^{\mathrm{f}}\left(t>t_{0}\right)=T_{9}^{0}$ in the case of constant asymptotic temperature (red line, see also left panel of Fig. 6 ). The other wind parameters are again the same as in Figs. $4-9\left(s=145, Y_{\mathrm{e}}=0.42, \tau_{\mathrm{dyn}}=5 \mathrm{~ms}\right)$. The right panel shows the distribution of cadmium isotopes at time $t=0.1 \mathrm{~s}$ for the values of $T_{9}^{\mathrm{f}}\left(t_{0}\right)$ listed in the inset; the neutron number densities at $t=0.1 \mathrm{~s}$ are given, too (cf. Fig. 8). The plot should be compared with the right panel of Fig. 6.

between (n, $\gamma$ )-reactions and $\beta$-decays. The nucleosynthesis at these conditions resembles the n-process of Blake \& Schramm (1976), but there it was discussed to occur because of a decrease in the neutron density below $10^{18} \mathrm{~cm}^{-3}$, while here it happens because of a decrease in the temperature and an associated strong reduction of the $(\gamma, \mathrm{n})$-rates. It should be noted that the prominent odd-even effect in the isotope distributions in the right panel of Fig. 6 for $T_{9}^{0}=1.4$ and 0.4 has practically disappeared in Fig. 10, where the isotope distributions for the same values of the transition temperature are much smoother.

We point out here that the r-processing of heavy nuclei through a quasi-equilibrium of (n, $\gamma$ )-reactions and $\beta$-decays at conditions where photodisintegrations are practically unimportant was recently also discussed by Wanajo (2007), who coined the term "cold r-process" 3 . We prefer to call it " $\mathrm{r} \beta$-process", because this name is conform with the denotation of other processes (r-process, rp-process, $v$ p-process,...) and reflects the essential aspect that characterizes this variant of the rapid neutron-capture process.

\subsection{Variations of wind parameters in the exponential phase}

In this section we will discuss the sensitivity of our nucleosynthesis results to variations of the characteristic outflow conditions like exponential expansion timescale, entropy, and electron fraction. Table 1 lists corresponding parameter values for some of the considered outflows: $\tau_{\text {dyn }}$ is the exponential expansion timescale ${ }^{4}, T_{9}^{\mathrm{f}}\left(t_{0}\right)$ and $t_{0}$ the temperature and time at which the transition occurs from the exponential first expansion

3 Different from us, Wanajo (2007) used neutrino-driven wind trajec-
tories obtained as solutions of the steady-state wind equations, and as-
sumed the transition to a constant freeze-out temperature $T_{\mathrm{f}}$ at some
radius. As argued in Sect. 2.1 , such a more realistic description of the
early outflow dynamics in combination with constant conditions at late
times shares some basic features with the simple two-stage expansion
behavior considered in our work.
4 We stress that comparing our expansion timescale with those given
in other papers requires some caution. Otsuki et al. (2000), Sumiyoshi
et al. (2000), and Terasawa et al. (2002) defined the expansion timescale
as $e$-folding time at $T=0.5 \mathrm{MeV}$, which is compatible with our use. In

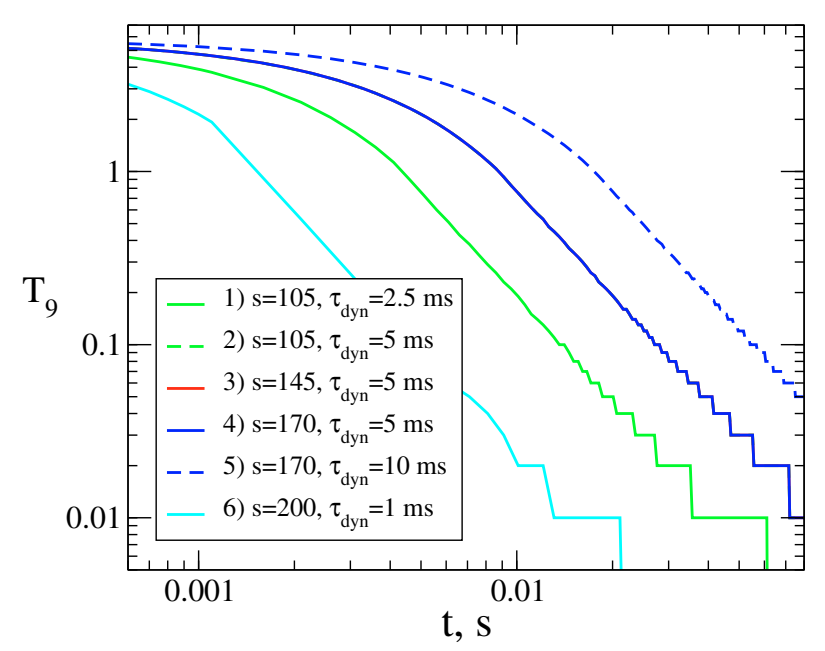

Fig. 11. Time evolution of the temperature for the different expansion histories considered in Fig. 12. All have an exponential first expansion phase and second stage of power-law decline. Note that the lines coincide for cases with different entropies $s$ but the same expansion timescale $\tau_{\text {dyn }}$.

phase to the second phase with power-law decline of temperature and density (the temperature evolution for different considered cases is displayed in Fig. 11$), v_{\text {ini }}=R_{\text {ini }} / \tau_{\text {dyn }}$ is the initial outflow velocity at an assumed initial radius $R_{\text {ini }}=10 \mathrm{~km}$, $v_{0}=v_{\text {ini }} \exp \left(t_{0} / \tau_{\text {dyn }}\right)$ is the outflow velocity at transition time $t_{0},\langle A\rangle$ is the average mass number of nucleosynthesis yields, and $\left\langle Y\left(A_{3}\right) / Y\left(A_{2}\right)\right\rangle$ the ratio of the height of the third abundance peak relative to the second as defined in Sect. 3.1.

As discussed in Sect. 2, the strength of the r-process is determined by the abundance of seed nuclei that is able to form, and by the number of remaining free neutrons when the r-processing sets in at $T_{9} \approx 2-3$. For the homologous outflows considered there we saw that a larger dynamical timescale increases the efficiency of seed formation at the expense of free neutrons, and for

contrast, Wanajo et al. $(2001,2002)$ defined it as the cooling time from $T=0.5 \mathrm{MeV}$ to $T=0.2 \mathrm{MeV}$, which is about $10 \%$ smaller. 

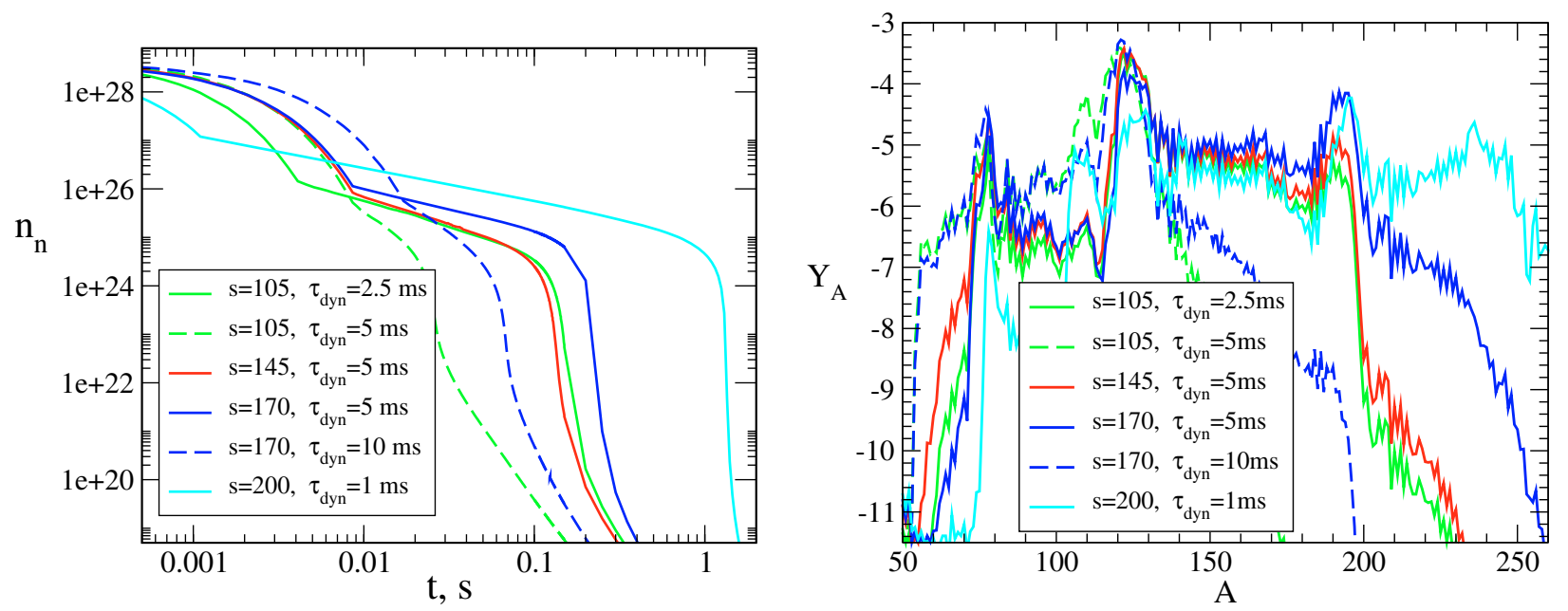

Fig. 12. Time evolution of the free neutron density (left) and final abundance distributions of r-process nuclei (right) for outflows with different assumed entropies $s$ and exponential expansion timescales $\tau_{\mathrm{dyn}}$ as listed in the inset. Different entropy values are associated with different line colors, a variation of the expansion timescale with dashed lines. In all cases we assumed $Y_{\mathrm{e}}=0.42$ and a transition temperature of $T_{9}^{\mathrm{f}}\left(t_{0}\right)=1$ between the exponential first expansion phase and the second phase of power-law temperature decline. The corresponding temperature evolution is plotted in Fig. 11. The cyan line denotes the most extreme considered case with an entropy of $s=200$ and a dynamical timescale of $\tau_{\text {dyn }}=1 \mathrm{~ms}$. It serves for demonstrating the influence of fission cycling (see also Fig. 14).

a very short dynamical timescale the expansion can be so rapid that $\alpha$ particles and neutrons do not have any time to assemble to very heavy elements. In both cases a strong third abundance peak cannot develop. A lower value of the outflow entropy also allows neutrons, protons, and $\alpha$-particles to recombine to nuclei more efficiently and therefore lower entropies have a similar effect as a slow expansion.

For a given value of the entropy, significant production of high-mass elements therefore on the one hand requires that the expansion timescale is sufficiently long, corresponding to a critical lower limit $Y_{\mathrm{s}}^{\mathrm{cr}}$ of the seed abundance at the start of the r-processing. On the other hand, the expansion must be enough fast because otherwise the seed production exceeds $Y_{\mathrm{s}}^{\mathrm{cr}}$ too much, and the platinum peak cannot be formed because of a disfavorably low neutron-to-seed ratio. Our systematic runs for homologous outflows showed (see Fig. 2) that with an entropy of $s=145$ and an electron fraction of $Y_{\mathrm{e}}=0.42$ only exponential timescales $\tau_{\text {dyn }}$ of less than about $10 \mathrm{~ms}$ lead to a neutron number fraction of $Y_{\mathrm{n}} \gtrsim 0.05$ and thus to neutron-to-seed ratios around 20 at the beginning of the r-process. Only then the nuclear flow has a chance to go beyond a mass number of 130 and to reach the range of $A \sim 196$, although the third peak may still be significantly underabundant compared to solar values (see below). The critical limit for the seed abundance turned out to be $Y_{\mathrm{s}}^{\mathrm{cr}} \sim 0.003$ (cf. Sect. 2). Of course, this number depends on $s$ and $Y_{\mathrm{e}}$, and the homologous expansion timescale that enables third peak formation is shorter for lower entropy values.

Let us now discuss the case in which the homologous outflow with its exponential density and temperature decline is replaced by a slower power-law temperature decay during the late expansion stage, i.e. after the freeze-out of charged-particle reactions. This makes strong r-processing up to the platinum peak possible for a much wider range of dynamical timescales $\tau_{\text {dyn }}$ than in the case of purely homologous evolution, practically for all values below some upper limit. This can be seen in Fig. 12, which displays the time-evolution of the neutron number density and the final abundance distribution for different choices of entropies and dynamical timescales. The second stage of powerlaw temperature decline is assumed to set in at $t=t_{0}$ with a transition temperature of $T_{9}^{\mathrm{f}}\left(t_{0}\right)=1$. A pronounced platinum peak develops for $s=105$ if $\tau_{\text {dyn }} \lesssim 2.5 \mathrm{~ms}$, for $s=145$ this needs $\tau_{\text {dyn }} \lesssim 5 \mathrm{~ms}$, and for $s \approx 170-200$ it requires $\tau_{\text {dyn }} \lessgtr 10 \mathrm{~ms}$. An extreme case with $s=200$ and $\tau_{\text {dyn }}=1$ ms leads to fission cycling and demonstrates that even for very fast homologous expansion during the first stage the slower evolution in the second phase allows all neutrons to be captured into heavy nuclei. The dashed lines belong to cases where the expansion in the homologous phase is too slow for third peak formation.

Figure 12 demonstrates that for a wide range of timescaleentropy combinations in the considered intervals, $2.5 \mathrm{~ms} \lesssim$ $\tau_{\text {dyn }} \lesssim 10 \mathrm{~ms}$ and $100 \lesssim s \lesssim 200$, heavy r-process elements up to the third abundance peak and beyond can be produced. Inspecting our results we find that a very strong platinum peak appears for conditions that roughly fulfill the relation $s \gtrsim 10\left(\tau_{\text {dyn }}+10\right)$.

In Fig. 13 the sensitivity of the r-processing to variations of $Y_{\mathrm{e}}$ is shown in the case with $s=170$ and $\tau_{\text {dyn }}=5 \mathrm{~ms}$. In the left panel one can see that a change in $Y_{\mathrm{e}}$ from 0.42 to 0.46 leads to a reduction of $n_{\mathrm{n}}$ at the beginning of the r-process and a more rapid exhaustion of free neutrons. The yields beyond the second peak are correspondingly lower, with a growing discrepancy at higher mass numbers $A$ (right panel of Fig. 13. While for $Y_{\mathrm{e}}=0.42$ the platinum peak is slighly overabundant compared to observations (see below), increasing $Y_{\mathrm{e}}$ by about $10 \%$ to 0.46 is enough to lead to a significant underproduction. For the considered short dynamical timescale this can be compensated by a roughly $30 \%$ higher value of the outflow entropy.

In Fig. 14 we provide an overview of the platinum peak formation in dependence of the entropy and dynamical timescale of the model outflows, using $Y_{\mathrm{e}}=0.42$ and assuming a transition temperature of $T_{9}^{\mathrm{f}}\left(t_{0}\right)=1$ between the exponential first cooling phase and the second phase with power-law decrease in temperature and density. The left panel confirms what we described above: for higher values of the entropy the $A \sim 196$ peak can be assembled for an increasingly wider range of expansion timescales. The bold horizontal lines in the left panel mark the observational band for the abundance ratio $\left\langle Y\left(A_{3}\right) / Y\left(A_{2}\right)\right\rangle$. The third peak relative to the second tends to become overabundant 

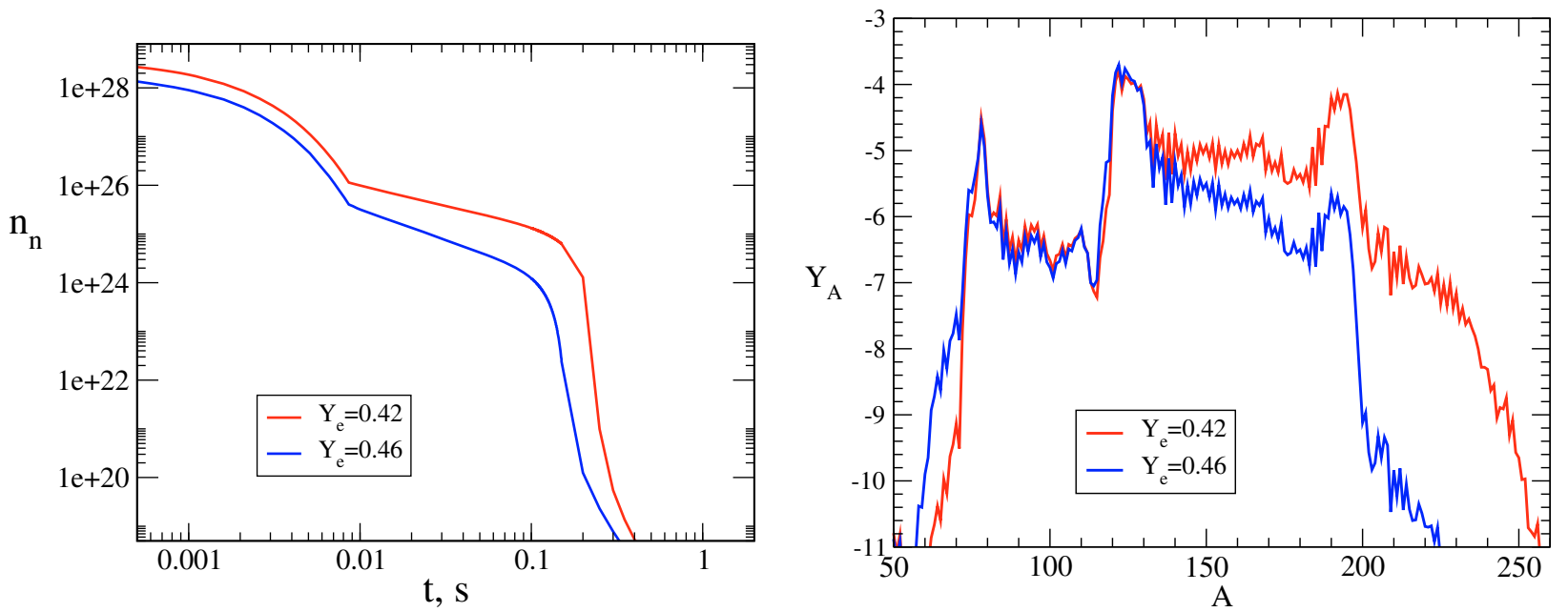

Fig. 13. Time-evolution of the free neutron number density $(l e f t)$ and final abundance distribution of r-process nuclei $(r i g h t)$ for $Y_{\mathrm{e}}=0.42$ (red lines) compared to $Y_{\mathrm{e}}=0.46$ (blue lines). In both cases we used $s=170, \tau_{\mathrm{dyn}}=5 \mathrm{~ms}$, and a transition temperature of $T_{9}^{\mathrm{f}}\left(t_{0}\right)=1$ between the exponential first expansion phase and the second phase of power-law temperature decline.
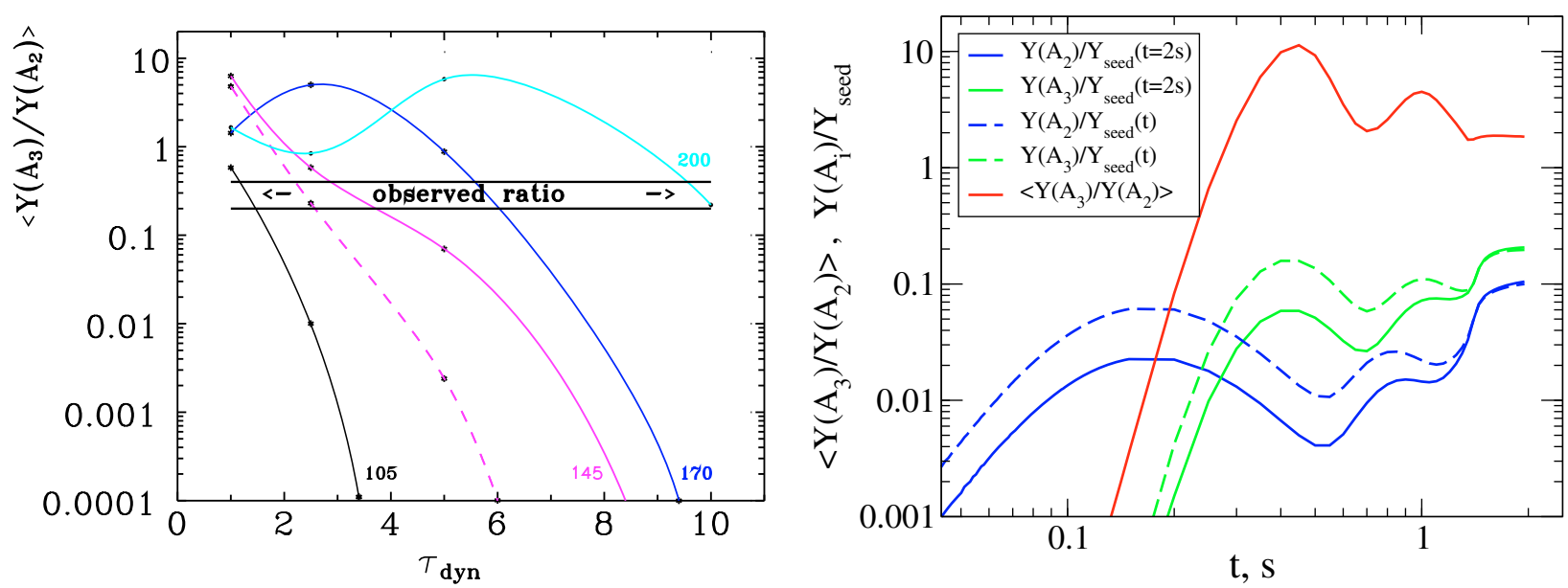

Fig. 14. Left: height of the third abundance peak relative to the second as function of the exponential expansion timescale $\tau_{\mathrm{dyn}}$, for different values of the entropy (labels at curves). The solid lines correspond to outflows with a power-law temperature decay following the exponential expansion, while the dashed line shows for comparison the results for $s=145$ and constant temperature $T_{9}^{\mathrm{f}}\left(t>t_{0}\right)=T_{9}^{0}$ during the second, slow expansion phase. In all cases the outflow was assumed to have an electron fraction of $Y_{\mathrm{e}}=0.42$ and the transition temperature was taken to be $T_{9}^{\mathrm{f}}\left(t_{0}\right)=1$. The band between the two bold horizontal lines marks the observational range. Right: time evolution of the ratio of the third abundance peak to the second peak and of the second and third abundance peaks, $Y\left(A_{2}\right)$ and $Y\left(A_{3}\right)$ (according to the definition in Eq. (11)), normalized to the total amounts of seed nuclei, $Y_{\text {seed }}$, at any given time $t$ (dashed lines) and to the seed abundance at the end of the evolution at $t=2 \mathrm{~s}$ (solid lines), respectively; the different line styles and colors are labelled in the inset box. Note that we consider all nuclei with $Z>2$ as seeds. The results were computed for $s=200, \tau_{\mathrm{dyn}}=1 \mathrm{~ms}, Y_{\mathrm{e}}=0.42$, and $T_{9}^{\mathrm{f}}\left(t_{0}\right)=1$. The waves visible in the time evolution of the different quantities reflect the effects of fission cycling in an environment with a very high free neutron density, which is the case for the extreme parameters of this run.

compared to the observations when the expansion timescale is low, whereas it remains too weak for long expansion timescales.

The dashed line in the left panel of Fig. 14 corresponds to runs with $s=145$ where a constant density and temperature were adopted during the second phase instead of the power-law behavior. The differences between the dashed and solid lines for the same entropy are large, in particular for longer dynamical timescales. This demonstrates again the importance of the latetime behavior of the outflow. This importance, however, is significantly reduced when the transition temperature $T_{9}^{\mathrm{f}}\left(t_{0}\right)$ is chosen to be near 0.4 (see the left panel of Fig. 10).

For entropies near 200 or higher fission cycling can occur. The right panel of Fig. 14 shows the time evolution of the second and third abundance peaks and of the ratio between both for a case with $s=200, \tau_{\text {dyn }}=1 \mathrm{~ms}$, and $Y_{\mathrm{e}}=0.42$. The periodic changes in the abundances and of $\left\langle Y\left(A_{3}\right) / Y\left(A_{2}\right)\right\rangle$ reflect the repeated propagation of nucleosynthesis wave through the transuranium region via fission cycling (the number of nuclear species is increased roughly by a factor of three due to fission), similar to what was observed by Panov et al. (2003), Goriely et al. (2005), and Beun et al. (2008). The overabundance of the platinum peak in this case depends significantly on the nuclear data Panov et al. (2001b) and should be explored separately on the basis of new fission data calculations (Panov et al. 2005).

\section{Discussion and conclusions}

Focussing on the high-entropy neutrino-driven wind environment, we investigated the sensitivity of r-process nucleosynthesis to the asymptotic low-temperature behavior that is assumed to follow an approximately homologous early expansion of very 
rapidly accelerated outflows. On the one hand we compared the continuously homologously evolving case to a situation where the temperature and density reach a lower limit and remain constant later on. The latter setting is equivalent to the choice of a freeze-out temperature for supersonic wind solutions by Wanajo et al. (2002) and Wanajo (2007), and it is similar to the use of a constant value for the outer boundary pressure of subsonic breeze solutions as adopted by Sumiyoshi et al. (2000), Terasawa et al. (2002), and Wanajo et al. (2001). Breeze solutions, however, are characterized by the very important feature that they imply a causal connection between the physical conditions at the neutron star and at the outer boundary, i.e. the mass-loss rate and asymptotic temperature are not independent, whereas supersonic outflows with a limiting temperature are not subject to such a constraint. The constant conditions at late times correspond to a situation where the homologous outflow is asymptotically decelerated to very small (zero) velocity. This can be considered as a reasonably good representation of the situation when a supersonic wind is slowed down in a termination shock at colliding with the preceding supernova ejecta. On the other hand we investigated a case where the velocity was assumed to make a transition from the linear radial growth of the homologous phase to an asymptotically constant, nonvanishing value. In this case the density was assumed to decrease with time like $t^{-2}$ and the temperature (for constant radiation entropy) like $t^{-2 / 3}$. This is supposed to be a model for the situation when a supersonic wind reaches a second stage of slower acceleration at late times.

We have found that for given and constant wind radiation entropy (we explored $s \sim 100-200 k_{\mathrm{B}}$ per nucleon), electronto-baryon ratio $\left(Y_{\mathrm{e}} \sim 0.42-0.46\right)$, and (sufficiently small) wind expansion timescale $\left(\tau_{\text {dyn }} \sim 1-10 \mathrm{~ms}\right)$, a strong r-process with production of the third abundance peak depends not only on the value of the transition temperature between the two expansion phases, but also on the evolution of temperature and density during the second, slower stage. In the case of a constant asymptotic temperature and density, the formation of the platinum peak is enabled when the asymptotic temperature value is moderately high $\left(T_{9}^{0} \sim 0.2-0.8\right)$. When leaving this range towards lower or higher asymptotic temperatures, the possibility of third-peak formation strongly decreases. This behavior was also seen by Wanajo et al. (2002) and Terasawa et al. (2002) for, as they called them, "freeze-out temperatures" or "boundary temperatures" above $T_{9}^{0} \sim 0.4$, but the variation with even lower temperatures remained unexplored in both works.

In our second considered case with power-law decline of $T(t)$ and $\rho(t)$, the strength of the $A \sim 195$ abundance peak compared to the $A \sim 130$ peak turned out to be relatively independent of the transition temperature $T^{\mathrm{f}}\left(t_{0}\right)$ in the interval between about $3 \times 10^{8} \mathrm{~K}$ and $1.4 \times 10^{9} \mathrm{~K}$. For lower transition temperatures the third-peak formation decreases steeply and behaves similar to the case with constant asymptotic temperature and density. This means that the appearance and disappearance of a prominent platinum peak when the constant asymptotic temperature is lowered from $T_{9}^{0} \sim 1$ to $T_{9}^{0} \sim 0.1$, is not observed in the case of a power-law decline of temperature and density during the late wind evolution. Interestingly, both considered cases of late-time expansion behavior also lead to distinctive differences in the abundances of neighboring isotopes of r-process elements. For the power-law time-dependence not only more neutron-rich isotopes are formed but the isotopic distribution is also smoother.

Strong r-processing naturally requires a sufficiently fast expansion in the homologous phase so that charged-particle reactions freeze out before excessive seed production occurs and high neutron-to-seed ratios cannot be reached. Moreover, a relatively rapid temperature decline during the second, slower expansion phase, and at the same time a persistence of sufficiently high neutron number densities $\left(n_{\mathrm{n}} \gtrsim 10^{24} \mathrm{~cm}^{-3}\right)$ are favorable for driving the nuclear flow beyond the second abundance peak. For fixed radiation entropy $s$ as assumed in our models, temperature and density are coupled by the relation $s \propto \rho / T^{3}=$ const. In this case the asymptotic power-law decrease in both quantities enables third peak formation for a wider range of transition temperatures.

Terasawa et al. (2002) argued that lower asymptotic temperatures reduce the charged-particle reactions, which leads to less seed production and a higher neutron-to-seed ratio, thus causing a better agreement of the r-process yields with the solar abundances. For our calculations, which are based on the idealized two-stage model of the wind expansion, this argument cannot be made. Long before the corresponding asymptotic temperatures (between $T_{9}=0.1$ and $T_{9}=1.4$ ) are reached, namely already above $T_{9} \approx 2$, charged-particle reactions become inefficient because of the impenetrability of the nuclear Coulomb barrier for low-energy thermal protons. Instead, the described nucleosynthesis results can be understood by the density and temperature dependence of neutron-captures and nuclear photodisintegration reactions.

Since the $(\gamma, n)$-rates decrease steeply with falling temperature, their role diminishes with a lower transition temperature. For sufficiently high neutron densities the still rapid neutron captures force the r-process path towards the neutron-drip line, where the $\beta$-decay rates are high. Instead of going through a $(\gamma, \mathrm{n})-(\mathrm{n}, \gamma)$ equilibrium, the r-processing proceeds now as a quasi-equilibrium of $(\mathrm{n}, \gamma)$ reactions and $\beta$-decays, which suggests the term " $\mathrm{r} \beta$-process". This sitation in outflows that expand supersonically and cool quickly to a few $10^{8} \mathrm{~K}$ was recently also discussed by Wanajo (2007), who named it "cold r-process". The formation of the third abundance peak is possible in this situation, provided the neutron-to-seed ratio is high enough, because the $\beta$-decays are fast and allow for a quick assembling of heavy nuclei. In contrast, if the exponential expansion makes a transition to a constant temperature that is high, $(\gamma, \mathrm{n})$-reactions drive the r-process path towards the valley of stability where the $\beta$ decay rates are low and the r-processing therefore slows down. In this case the free neutrons are used up in forming the second abundance peak before any significant third maximum can build up. On the other hand, the power-law temperature decline in the slow expansion phase leads to an efficient $\mathrm{r} \beta$-process for a wide range of transition temperatures $T_{9}^{\mathrm{f}}\left(t_{0}\right)$. Because the strength of the third abundance peak then depends only on the neutron density, it becomes relatively insensitive to the value of $T_{9}^{\mathrm{f}}\left(t_{0}\right)$ between about 0.3 and 1.4. If the transition temperature is even lower, the free neutron density during the slow expansion phase of the outflow is not large enough any more to support a strong r-processing.

In previous investigations (Terasawa et al. 2001, 2002; Wanajo et al. 2002) the complex influence of the late-time outflow behavior was not explored, but it matters when the robustness of abundance yields to variations in the neutrino-driven wind models is discussed, especially in situations where high entropies, low temperatures, and high neutron densities lead to a change in the r-process to an $\mathrm{r} \beta$-process.

In summary, we therefore conclude that the detailed cooling behavior during the late expansion of supernova outflows can have important consequences for r-process nucleosynthesis. In particular, a slow expansion phase with decreasing temperature and density following a rapid, supersonic initial outflow expansion, is more favorable for a strong r-process than the 
constant boundary conditions assumed previously (e.g., Wanajo et al. 2001, 2002; Terasawa et al. 2001, 2002). Despite differences in the initial neutron number density by more than two orders of magnitude, such conditions can lead to a fairly robust production of the platinum peak for a wider range of transition temperatures (at least for the cases of very fast early expansion and modest values of entropy and neutron excess as considered in our study). This is caused by a kind of self-regulation of the quasi-equilibrium of $(\gamma, \mathrm{n})$-reactions and $\beta$-decays in the $\mathrm{r} \beta$-process, which leads to roughly the same number of neutron captures per seed nucleus, independent of whether the process proceeds quickly for high neutron densities (in which case the r-process path is closer to the neutron-drip line) or slower in the case of low neutron densities. This might give a hint for the outflow dynamics of neutrino-driven wind ejecta that can enable the uniform production of heavy r-process elements suggested by the abundance patterns observed in metal-poor stars (see e.g., Cowan \& Sneden 2006).

For the explored range of parameter values, the r-processing can last 150-200 ms or even longer. This duration is the lower time limit for the formation of heavy elements from iron-group seeds up to the platinum peak. For entropies $s$ between 100 and $200 k_{\mathrm{B}}$ per nucleon, a solar-like production of the heaviest elements can occur only in the case that the outflow expansion decelerates and a transition from the initial homologous phase to a slower later stage of expansion occurs. In addition, a short or very short exponential timescale $\left(\tau_{\text {dyn }} \sim 10 \mathrm{~ms}\right.$ for $s=200$ and $\tau_{\text {dyn }} \sim 1 \mathrm{~ms}$ for $\left.s=100\right)$ is needed during the homologous expansion, if moderately neutron-rich conditions $\left(Y_{\mathrm{e}}=0.42\right)$ are considered. Of course, if the neutron excess decreases, the formation of the third abundance peak requires a higher entropy. These parameter constraints are rather similar to those shown in Fig. 1 of Hoffman et al. (1997). This can be understood from the fact that supersonic solutions of the neutrino-driven wind equations as those employed by Hoffman et al. (1997) show approximately homologous expansion $(v \propto r)$ only up to some maximum radius, above which the velocity continues to grow much less rapidly. Although there is no deceleration as it is caused by a wind termination shock (see Arcones et al. 2007), the slow-down of the acceleration of supersonic winds has still some basic similarity to the transition from a rapid first expansion phase to a slower second stage as assumed in our simplified outflow models.

Acknowledgements. We are grateful to an anonymous referee for very valuable suggestions for improving our presentation. This work was in part supported by the SNF project No. IB7320-110996 and by the Deutsche Forschungsgemeinschaft through the Transregional Collaborative Research Centers SFB/TR 27 "Neutrinos and Beyond" and SFB/TR 7 "Gravitational Wave Astronomy", and through the Cluster of Excellence EXC 153 "Origin and Structure of the Universe" (http: //www . universe-cluster.de).

\section{References}

Arcones, A., Janka, H.-Th., \& Scheck, L. 2007, A\&A, 467, 1227

Arnould, M., Goriely, S., \& Takahashi, K. 2007, Phys. Rep., 450, 97

Beun, J., McLaughlin, G. C., Surman, R., \& Hix, W. R. 2008, Phys. Rev. C, 77, 035804

Bisnovatyi-Kogan, G. S., \& Chechetkin, V. M. 1979, Sov. Uspehi Phys. Nauk, 127,263

Blake, J. B., \& Schramm, D. N. 1976, ApJ, 209, 846

Blinnikov, S. I., \& Panov, I. V. 1996, Astron. Lett., 22, 39

Buras, R., Janka, H.-Th., Rampp, M., \& Kifonidis, K. A\&A, 2006, 457, 281

Burbidge, G. R., Burbidge, E. M., Fowler, W. A., \& Hoyle, F. 1957, Rev. Mod. Phys., 29, 547
Burrows, A., Hayes, J., \& Fryxell, B. A. 1995, ApJ, 450, 830

Cameron, A. G. W. 2003, ApJ, 587, 327

Cameron, A. G. W., Cowan, J. J., \& Truran, J. W. 1983, Ap\&SS, 91, 235

Cardall, C. Y., \& Fuller, G. M. 1997, ApJ, 486, L111

Cowan, J. J., \& Sneden, C. 2006, Nature, 440, 1151

Cowan, J. J., Thielemann, F.-K., \& Truran, J. W. 1991, Phys. Rep., 208, 267

Freiburghaus, C., Rosswog, S., \& Thielemann, F.-K. 1999, ApJ, 525, L121

Gear, C. W. 1971, Numerical Initial Value Problems in Ordinary Differential Equations (Englewood Cliffs, New Jersey: Prentice-Hall)

Goriely, S., \& Arnould, M. 1996, A\&A, 312, 327

Goriely, S., Demetriou, P., Janka, H.-Th., Pearson, J. M., \& Samyn, M. 2005, Nucl. Phys. A, 758 587c

Hillebrandt, W. 1978, Space Sci. Rev., 21, 639

Hoffman, R. D., Woosley, S. E., \& Qian, Y.-Z. 1997, ApJ, 482, 951

Imshennik, V. S. 1992, Astron. Lett., 18, 194

Imshennik, V. S., \& Litvinova, I. Yu. 2006. Atomic Nuclei, 69, 660

Janka, H.-Th., \& Müller, E. 1995, Phys. Rep., 256, 135

Janka, H.-Th., \& Müller, E. 1995, ApJ, 448, L109

Kratz, K.-L., Bitouzet, J.-P., Thielemann, F.-K., Möller, P., \& Pfeiffer, B. 1993, ApJ, 403, 216

Kuroda, T., Wanajo, S., \& Nomoto, K. 2008, ApJ, 672, 1068

Lattimer, J. M., \& Schramm, D. N. 1974, ApJ, 192, L145

Lattimer, J. M., \& Schramm, D. N. 1976, ApJ, 210, 549

Meyer, B. S., Mathews, G. J., Howard, W. M., Woosley, S. E., \& Hoffman, R. D. 1992, ApJ, 399, 656

Möller, P., Nix, J. R., Myers, W. D., \& Swiatecki, W. J. 1995, Atomic Data Nucl. Data Tables, 59, 185

Nadyozhin, D. K., \& Yudin, A. V. 2004, Astron. Lett., 30, 697

Nadyozhin, D. K., Panov, I. V., \& Blinnikov, S. I. 1998, A\&A, 335, 207.

Otsuki, K., Tagoshi, H., Kajino, T., \& Wanajo, S. 2000, ApJ, 533, 424

Panov, I. V. 2003, Astron. Lett., 29, 163

Panov, I. V., \& Chechetkin, V. M. 2002, Astron. Lett., 28, 476

Panov, I. V., \& Thielemann F.-K. 2003, Nucl. Phys. A, 718, 647

Panov, I. V., Blinnikov, S. I., \& Thielemann, F.-K. 2001a, Astron. Lett., 27, 1

Panov, I. V., Freiburghaus, C., \& Thielemann, F.-K. 2001b, Nucl. Phys. A, 688, 587

Panov, I. V., Kolbe, E., Pfeiffer, B., Rauscher, Th., Kratz, K.-L., \& Thielemann, F.-K. 2005, Nucl. Phys. A, 747, 633

Qian, Y.-Z., \& Wasserburg, G. J. 2000, Phys. Rep., 333, 77

Qian, Y.-Z., \& Woosley, S. E. 1996, ApJ, 471, 331

Rauscher, T., \& Thielemann, F.-K. 2000, Atomic Data Nucl. Data Tables, 75, 1

Rauscher, T., Thielemann, F.-K., \& Kratz, K.-L. 1997, Nucl. Phys., A 621, 331c.

Symbalisty, E. M. D., \& Schramm, D. N. 1982, ApL, 22, 143

Seeger, P. A., Fowler, W. A., \& Clayton, D. D. 1965, ApJS, 11, 121

Sumiyoshi, K., Suzuki, H., Otsuki, K., Terasawa, M., \& Yamada Sh. 2000, Publ. Astron. Soc. Japan., 52, 601

Takahashi, K., \& Janka, H.-T. 1997, Proc. of Int. Conf. Origin of Matter and Evolution of Galaxies in the Universe '96 Atami, Japan, 18-20 January 1996, ed. T. Kajino, Y. Yoshii, \& S. Kubono (World Scientific, Singapore), 213

Takahashi, K., Witti, J., \& Janka, H.-T. 1994, A\&A, 286, 857

Terasawa, M., Sumiyoshi, K., Kajino, T., Mathews, G. J., \& Tanihata, I. 2001, ApJ, 562, 470

Terasawa, M., Sumiyoshi, K., Yamada, S., Suzuki, H., \& Kajino, T. 2002, ApJ, 578, L137

Thielemann, F.-K., Arnould, M., \& Truran, J. W. 1987, in Advances in Nuclear Astrophysics, ed. E. Vangioni-Flam et al. (Gif-sur-Yvette: Éditions frontière) 525

Thompson, T. A., Burrows, A., \& Meyer, B. S. 2001, ApJ, 562, 887

Tomàs, R., Kachelrieß, M., Raffelt, G., et al. 2004, J. Cos. Astropart. Phys., 09, 015

Wanajo, S. 2007, ApJ, 666, L77

Wanajo, S., \& Ishimaru, Y. 2006, Nucl. Phys. A, 777, 676

Wanajo, S., Kajino, T., Mathews, G. J., \& Otsuki, K. 2001, ApJ, 554, 578

Wanajo, S., Itoh, N., Ishimaru, Yu., Nozawa, S., \& Beers, T. C. 2002, ApJ, 577, 853

Wanajo, S., Goriely, S., Samyn, M., \& Itoh, N. 2004, ApJ, 606, 1057

Witti, J., Janka, H.-T., \& Takahashi, K. 1994, A\&A, 286, 841

Witti, J., Janka, H.-Th., Takahashi, K., \& Hillebrandt, W. 1993, in Nuclei in the Cosmos-IX, ed. F. Käppeler, \& K. Wisshak (Bristol and Philadelphia: Inst. of Phys. Publ.), 601

Woosley, S. E., \& Hoffman, R. D. 1992, ApJ, 395, 202

Woosley, S. E., Wilson, J. R., Mathews, G. J., Hoffman, R. D., \& Meyer, B. S. 1994, ApJ, 433, 229 